\title{
MULTIPLICATIVE PROPERTIES OF POWER MAPS. II
}

\author{
BY
}

\author{
C. A. MCGIBBON ${ }^{1}$
}

\begin{abstract}
The notions of $A_{n}$-maps and $C_{n}$-forms can be regarded as crude approximations to the concepts of homomorphisms and commutativity, respectively. These approximations are used to study power maps on connected Lie groups and their localizations. For such groups the power map $x \mapsto x^{n}$ is known to be an $A_{2}$-map if and only if $n$ is a solution to a certain quadratic congruence. In this paper, $A_{3}$-power maps are studied. For the Lie group $\mathrm{Sp}(1)$ it is shown that the $A_{3}$-powers coincide with solutions which are common to the quadratic congruence, mentioned earlier, and another cubic congruence. Other Lie groups, when localized so as to become homotopy commutative, are also shown to have infinitely many $A_{3}$-powers. The proofs reflect the combinatorial nature of the obstructions involved.
\end{abstract}

1. Introduction, definitions and miscellanea. This paper continues the study of power maps on finite $H$-spaces that was started in part I [12]. Two considerations, which may not have been apparent in part I, prompted this investigation. The first consideration was the noncommutative nature of various Lie groups-even when viewed, up to homotopy, one prime at a time!

QUESTION 1. Let $G$ be a topological group with the homotopy type of a 1connected, finite $C W$ complex which is not contractible. To what extent does $G$, when localized at a prime $p$, fail to be commutative?

Before I explain the connection between this question and the study of power maps, I shall discuss in some detail the question itself. In attempting to answer it, there are two extreme cases that one should keep in mind. First, for any prime $p$, $G_{(p)}$ cannot be strictly commutative. This is an easy consequence of a result, due to J. C. Moore [15], that states that every abelian monoid has the weak homotopy type of a product of Eilenberg-Mac Lane spaces. The assumptions on $G$, and hence on $G_{(p)}$, clearly rule out this possibility.

The other extreme occurs when the prime $p=2$. Here, a celebrated theorem of J. R. Hubbuck [8] becomes applicable. This result is usually quoted as saying that the tori, $S^{1}, S^{1} \times S^{1}, \ldots$, are the only nontrivial examples of $H$-spaces which are connected, finite, and homotopy commutative. However, a glance at Hubbuck's proof, which uses complex $K$-theory localized at 2, reveals the 2-primary nature of

Received by the editors September 16, 1980 and, in revised form, June 24, 1981.

1980 Mathematics Subject Classification. Primary 55D45, 55D35, 55G37.

Key words and phrases. $A_{n}$-map, $C_{n}$-form, projective $n$-space, localization of spaces, homotopy commutativity.

${ }^{1}$ Supported in part by NSF. 
his result. For a finite $H$-space $X$, the rings $K\left(X, \mathbf{Z}_{(2)}\right)$ and $K\left(X_{(2)} ; \mathbf{Z}_{(2)}\right)$ are naturally isomorphic and so it follows that Hubbuck has answered Question 1 in the case $p=2$; namely, $G_{(2)}$ is not even homotopy commutative. For larger primes, however, this need not be the case; the Lie group $\mathrm{Sp}(1)$, for example, is homotopy commutative at the prime $p=5$. More generally, if $G=\operatorname{SU}(n)$ and $p>2 n$, then $G_{(p)}$ is homotopy commutative [13].

Thus to answer Question 1 for large primes $p$, we need to measure the noncommutativity of $G_{(p)}$ in terms other than homotopy commutativity. For this purpose I recall the definition of a $C_{n}$-form. These forms are implicit in the pioneering work of Sugawara [21], but the presentation given here follows that of F. D. Williams [24].

A $C_{2}$-form on a group $X$ is a commuting homotopy $T: X \times X \times I \rightarrow X$ such that $T(x, y, 0)=x y$ and $T(x, y, 1)=y x$. Notice that a $C_{2}$-form enables us to commute continuously, up to homotopy, two variables on $X$.

As might be expected, the definition of a $C_{3}$-form on $X$ involves three variables. We first use the $C_{2}$-form $T$ to define a map $C B_{3}(T): X \times X \times X \times S^{1} \rightarrow X$, whose adjoint sends

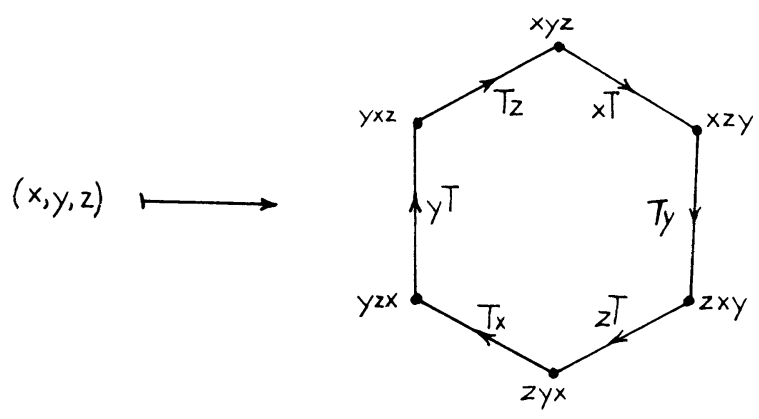

In this notation, the $B$ is meant to suggest boundary. If the map $C B_{3}(T)$ extends to $X^{3} \times D^{2} \rightarrow X$, then such an extension is called a $C_{3}$-form on $X$.

At this point it is easy to predict the general pattern; a $C_{n}$-form on $X$ should correspond to a solution of the extension problem for a map $C B_{n}: X^{n} \times S^{n-2} \rightarrow X$. The construction of the map $C B_{n}$, in turn, should involve all of the previously defined $C_{r}$-forms on $X$ as well as an unusual cell division of the sphere $S^{n-2}$. The $n$ ! vertices of this cell division correspond to the various permutations of the product $x_{1} x_{2} \cdots x_{n}$. The precise definitions and details can be found in [24].

The relevance of $C_{n}$-forms to Question 1 is demonstrated by the next result.

Proposition 2. Suppose that $G \simeq_{p} S^{2 n_{1}-1} \times \cdots \times S^{2 n_{r}-1}$ where $n_{1}>1$. Then there is no loop multiplication on $G_{(p)}$ which admits a $C_{n}$-form for all $n$.

Proof. By Hubbuck's theorem, this is obviously true if $p=2$. Assume then for some odd prime $p$ that $G_{(p)}$ has the homotopy type of a $C_{\infty}$-loop space. By Corollary 18 of [24] it follows that the double suspension $E^{2}: G_{(p)} \rightarrow \Omega^{2} \Sigma^{2} G_{(p)}$ has a left inverse. This and our hypothesis imply that $E^{2}: \pi_{*} S_{(p)}^{2 n_{1}-1} \rightarrow \pi_{*} \Omega^{2} S_{(p)}^{2 n_{1}+1}$ is injective. But this is a contradiction because for $n>1$ the double suspension is known to have a nonzero kernel in $\pi_{2 n p-3} S_{(p)}^{2 n-1}[\mathbf{1 1}, \mathbf{1 6}]$. 
Notice that this result answers Question 1 for all but a finite number of primes. In particular, for the regular primes $p$, it shows that $G_{(p)}$ is less commutative than is $\Omega B$, where $B$ is an $H$-space [24]. I suspect that the answer for the remaining primes is the same, but that it may be harder to prove.

The following simple exercise illustrates an algebraic connection between power maps and commutativity.

EXERCISE 3. Show that a group $G$ is abelian if and only if the power map $x \mapsto x^{n}$ on $G$ is a homomorphism for some three consecutive values of $n$.

This exercise has an analogue in homotopy theory. To state this analogue, we first need the definition of an $A_{n}$-map. Just as the notion of strict commutativity is successively approximated by the existence of a $C_{n}$-form, so is the concept of a continuous homomorphism approximated by that of an $A_{n}$-map. However, in both cases these approximations converge to homotopy theoretic notions which do not in general coincide with the group theoretic models.

The idea of an $A_{n}$-map first appeared in Sugawara's definition of a strongly homotopy multiplicative map [20]. The following treatment is due to Stasheff [18].

Let $f:(X, \cdot) \rightarrow(G,+)$ be a map between monoids (which are not necessarily commutative). We say that $f$ is an $A_{2}$-map if there is a homotopy $F: X \times X \times I \rightarrow G$ which assigns to each pair $(x, y) \in X^{2}$ the following path on $G$.

$$
F(x, y, 0)=f(x y) \bullet F(x, y) \longrightarrow f(x)+f(y)=F(x, y, 1) .
$$

There could be many choices for the $A_{2}$-homotopy $F$ (any two choices would differ by an element of [ $X \wedge X, \Omega G]$ ). We say that $f$ is an $A_{3}$-map if for some choice of $F$, the map $A B_{3}(F): X \times X \times X \times S^{1} \rightarrow G$, whose adjoint sends

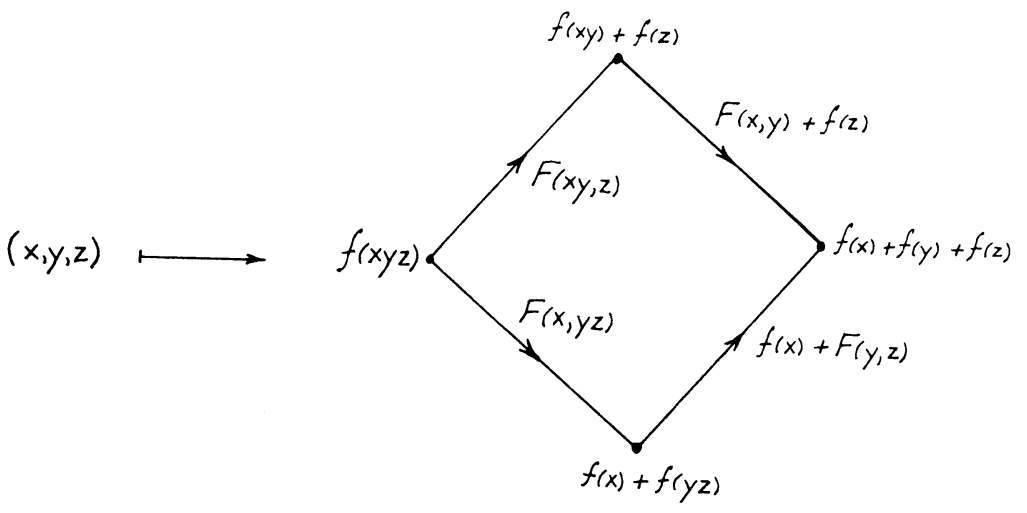

extends to $X^{3} \times D^{2} \rightarrow G$. Such an extension will be called an $A_{3}$-homotopy for $f$.

The general definition of an $A_{n}$-homotopy is now quite easy to predict and is very similar, in spirit, to that of $C_{n}$-form; likewise, the precise details are somewhat formidable (see [19, Chapter 11]).

In [12], I classified (in theory at least) those power maps which are $A_{2}$-maps (or $H$-maps) on certain finite $H$-spaces. The main result there was the following: 
THEOREM 4. Let $(X, \mu)$ be a homotopy associative $H$-space where $X$ has the homotopy type of a connected finite $C W$ complex (or a localization of such a complex). Then there is an integer $N=N(X, \mu) \geqslant 2$, such that the power map $x \mapsto x^{\lambda}$ is an $H$-map on $(X, \mu)$ if and only if $\lambda(\lambda-1) \equiv 0 \bmod N$.

As mentioned in [12], this number $N$ is a measure of the lack of homotopy commutativity on $(X, \mu)$. Moreover, $N(X, \mu)=2$ if and only if $(X, \mu)$ is homotopy commutative (or $C_{2}$ ). The following analogue of Exercise 3 is an immediate consequence of Theorem 4.

Corollary 4.1. If $(X, \mu)$ is an H-space as in Theorem 4 , then $(X, \mu)$ is $C_{2}$-space if and only if the power map $x \mapsto x^{n}$ is an $A_{2}$-map for some three consecutive values of $n$.

In this paper I push this analogy a bit further.

COROllary 9.1. Let $(X, \mu)$ be a loop space where $X$ satisfies the connectivity and finiteness requirements of Theorem 4. If, in addition, $[X \wedge X, \Omega X]=0$, then $(X, \mu)$ is a $C_{3}$-space if the power map $x \mapsto x^{n}$ is an $A_{3}$-map on $(X, \mu)$ for some four consecutive values of $n$.

These two analogues of Exercise 3 strike me as being more amusing than important. Nevertheless they do illustrate the close relationship between the study of power maps and questions of noncommutativity. This relationship was one of the considerations which led to this series of papers.

The other consideration was a search for examples. In the literature there are many examples of spaces which are $A_{n}$, in Stasheff's terminology, but not $A_{n+1}$. The simplest of these are localized spheres; for each prime $p, S_{(p)}^{2 p+1}$ is $A_{p-1}$ but not $A_{p}$ $[19$, p. 53]. Such examples show that in the homotopy category a theory of successive approximations, such as $A_{n}$-structures, is useful and is indeed necessary.

I wanted to find similar examples of maps. There is what might be considered the standard example.

THEOREM 5. Let $X$ be a monoid with the homotopy type of a connected, noncontractible $C W$ complex. Let $P_{n}(X)$ denote the projective $n$-space of $X$. For $1 \leqslant n<\infty$, the standard inclusion $X \rightarrow \Omega P_{n}(X)$ is an $A_{n}$-map but it is never an $A_{n+1}$-map.

Most of this theorem is due to Stasheff. In [19, Theorem 8.4] he showed that the map in question is always an $A_{n}$-map. He also showed that $X \rightarrow \Omega P_{n}(X)$ is not an $A_{n+1}$-map, when $X=S^{1}$. My contribution to Theorem 5 could be viewed as showing that the example just cited is not exceptional, but rather part of a general pattern.

While Theorem 5 certainly gives us many examples, it somehow misses the mark; it goes much further in describing projective $n$-space than it does in explaining the special nature of an $A_{n}$-map. It therefore seemed more meaningful to consider maps that arise more naturally on any topological group, namely, power maps.

For power maps on connected, compact Lie groups, Theorem 4 represents one extreme, the classification of $A_{2}$-power maps. The other extreme, $A_{\infty}$-power maps, is covered in the next two results. 
Historically, the first nontrivial example was the following:

THEOREM 6. The power map $x \mapsto x^{\lambda}$ on the Lie group $\mathrm{Sp}(1)$ is an $A_{\infty}$-map if and only if $\lambda$ is an odd square or zero.

The necessity of these arithmetic conditions has achieved a folklore status as many people have worked out their own proof; Feder and Gitler published theirs in [5]. The sufficiency was one of the early triumphs of localization and completion techniques and is due to Sullivan [22].

On the group $\mathrm{Sp}(1)$, the notion of a power map and that of an unstable Adams operation coincide up to homotopy. In most other cases they do not. This accounts for the disparity between Theorem 6 and the next result. The following theorem should be regarded as a corollary of Hubbuck's extensive results on self maps of classifying spaces [9].

THEOREM 7. Assume that $G$ is a compact, 1-connected Lie group with $\pi_{m}(G) \otimes \mathbf{Q} \neq 0$ for some $m>3$. Then the power map $x \mapsto x^{\lambda}$ is an $A_{\infty}$-map on $G$ if and only if $\lambda=0$ or 1 .

2. Statement of the main results. The main results in this paper deal with the existence and classification of those power maps which are $A_{3}$-maps on a connected topological group. The role of the first nontrivial example is again played by the Lie group $\mathrm{Sp}(1)$.

THEOREM 8. On $\mathrm{Sp}(1)$, the power map $x \mapsto x^{\lambda}$ is an $A_{3}$ map if and only if $\lambda(\lambda-1) \equiv 0 \bmod 24$ and $\lambda(\lambda-1)(\lambda-4) \equiv 0 \bmod 360$.

This result proves the case $n=3$ of a conjecture, due to Feder and Gitler [5], which concerns the homological classification of self maps of quaternionic projective $n$-space.

The next theorem, which is the main result, arose in an attempt to generalize Theorem 8 to other Lie groups. In view of Theorem 7, it is not obvious that, for such groups, there should be any $A_{3}$-powers besides 0 and 1 . The next two results do not rule out this possibility; they do, however, make it seem unlikely. In fact, they show that if we take a 1-connected Lie group $G$, and invert those primes at which $G$ is not homotopy commutative (i.e., those primes which divide the order of the basic 2-fold Samelson product on $G$ ) then the resulting localized, homotopy comutative group has infinitely many $A_{3}$-powers. Indeed, it has at least two distinct arithmetic sequences of such powers.

Let $T$ denote a $C_{2}$-form on a topological group $(X, \mu)$. The next theorem is stated in terms of three special properties of the triple $(X, \mu, T)$. To describe these properties, I shall use $T$ to define the adjoints of three maps of the form $L_{i}$ : $X \times X \times S^{1} \rightarrow Y(i=1,2,3)$. The $C_{2}$-space $(X, \mu, T)$ will then be said to have property $\mathrm{P}_{i}$ if the map $L_{i}$ extends to $X \times X \times D^{2} \rightarrow Y$. Here are the maps.

$$
L_{1}\left\{\begin{array}{l}
X \times X \times S^{1} \rightarrow X \\
(x, y) \mapsto T(x, y) \oplus T(y, x)
\end{array}=x y<y x\right.
$$


A space with property $\mathrm{P}_{1}$ is easily seen to be an $H_{2}$-space in the sense of Kudo and Araki (cf. [19, p. 81]).

$$
L_{2}\left\{\begin{array}{l}
X \times X \times S^{1} \rightarrow X \\
(x, y) \mapsto T^{-1}(x, y) \oplus T\left(x^{-1}, y^{-1}\right)
\end{array}=(x y)^{-1} T_{(x, y)}^{-1}>(y x)^{-1}\right.
$$

The third map requires additional notation and assumptions. Assume that the rationalization ( $X_{0}, \mu_{0}$ ) is $A_{3}$-equivalent to strictly commutative group, which will be denoted as $\left(X_{0},+\right)$. Regard the rational equivalence $X \rightarrow X_{0}$ as an embedding and let $R: X^{2} \times I \rightarrow X_{0}$ be an $A_{2}$-homotopy between $x y$ and $x+y$, such that the $A_{3}$-obstruction $A B_{3}(R)$ extends to $X^{3} \times D^{2} \rightarrow X_{0}$. The map $L_{3}$ is then defined as follows:

$$
\begin{aligned}
L_{3}\left\{\begin{array}{l}
X \times X \times S^{1} \rightarrow X_{0} \\
(x, y) \mapsto \theta R(x, y) \oplus T(x, y) \oplus R(y, x)
\end{array}\right. \\
=\underbrace{}_{x y} \underbrace{R x}_{R(x, y)} x+y=y+x
\end{aligned}
$$

Let $G$ be a homotopy commutative group. For each integer $n$, there is a set of obstructions to the power map $x \mapsto x^{n}$ being an $A_{3}$-map on $G$. The problem is, of course, to determine when the trivial class is in this set of obstructions. With the aid of the three properties just described, the following theorem gives a partial solution to this problem.

THEOREM 9. Let $(X, \mu, T)$ be a $C_{2}$-topological group with the homotopy type a connected $C W$ complex. For each integer $n$, there is an element $\Phi(n)=\Phi(n, T) \in[X$ $\wedge X \wedge X, \Omega X]$ such that if $\Phi(n)=0$, then the power map $x \mapsto x^{n}$ is an $A_{3}$-map on $(X, \mu)$. Moreover

(i) If $T$ satisfies property $\mathrm{P}_{1}$, then for all $n \geqslant 0$,

$$
\Phi(n)=\frac{n(n-1)}{2} \Phi(2)+\frac{n(n-1)(n-2)}{6} B_{3}(T) .
$$

(ii) If $T$ also satisfies property $\mathrm{P}_{2}$, then the formula in (i) also holds for all negative integers.

(iii) If $T$ satisfies properties $\mathrm{P}_{1}$ and $\mathrm{P}_{3}$, then the classes, $\Phi(2)$ and $B_{3}(T)$ have finite order in the group $[X \wedge X \wedge X, \Omega X]$. 
It is easy to see in Theorem 9 that the polynomial relation between the obstructions in part (i) and the finiteness of their orders in part (iii) imply the existence of infinitely many $A_{3}$-powers for a group with these properties.

Two other items in Theorem 9 require some explanation. The obstruction element $\Phi(n)$ depends not only on the $C_{2}$-form $T$ but also on a rather peculiar $A_{2}$-homotopy between $(x y)^{n}$ and $x^{n} y^{n}$ which is constructed using $T$. See $\S 5$ for details. Secondly, the class $B_{3}(T)$, introduced in the statement of Theorem 9 , is the normalized adjoint of the "hexagon" $C B_{3}(T)$ that was defined in $\$ 1$. Again precise details are given in $\$ 5$.

Here are some examples of groups to which Theorem 9 can be applied.

THEOREM 10. (i) Let $X$ be a homotopy commutative group with the homotopy type of a connected $C W$ complex. If the power map $x \mapsto x^{2}$ is a homotopy self-equivalence of $X$, then there is a $C_{2}$-form on $X$ with properties $\mathrm{P}_{1}$ and $\mathrm{P}_{2}$.

(ii) Let $X=G_{(l)}$ where $G$ is a compact 1-connected Lie group and $l$ is a set of primes at which $G$ is homotopy commutative. Then there is a $C_{2}$-form $T$ on $X$ such that

(a) Thas properties $\mathrm{P}_{1}$ and $\mathrm{P}_{2}$, and

(b) the classes $\Phi(2, T)$ and $B_{3}(T)$ have finite order.

(iii) For each prime $p>3$, let $Y_{p}$ be a topological group with the homotopy type of $S_{(p)}^{p-2}$. By Sullivan [22, p. 4.28] such a group exists. Then, up to homotopy, there is a unique $C_{2}$-form on $Y_{p}$ with properties $\mathrm{P}_{1}, \mathrm{P}_{2}$, and $\mathrm{P}_{3}$. Moreover, the power map $y \mapsto y^{n}$ is an $A_{3}$-map on $Y_{p}$ if and only if $n^{3}-n \equiv 0 \bmod p$.

3. Organization, notation, and acknowledgements. Theorems 5, 7 and 8 are proved in \$4. The proofs of these results employ the standard machinery and notation of algebraic topology. The proofs of Theorems 9 and 10 are given in $\S 5$. The combinatorial nature of these proofs requires much special notation. This notation is introduced and explained as it is needed, usually with the aid of a picture. The last section, $\S 6$, consists of open questions related to this work.

Most of the results in this paper are stated in terms of topological groups. In particular if $G$ is a topological group, then the localization $G_{(l)}$ is also assumed to be a topological group. To be precise, $G_{(l)}$ will denote any topological group which is $A_{\infty}$-equivalent to the loop space $\Omega\left((B G)_{(l)}\right)$. A specific model for $G_{(l)}$ can be obtained using Milnor's construction [14] or Kan's free simplicial group construction $[10]$.

I would like to thank my advisor Sufian Husseini for sharing with me the intuition which eventually led to the proof of the main result, Theorem 9. I would also like to thank Jim Stasheff for his interest and encouragement, and for his lovely book [19].

\section{Proofs.}

Proof of Theorem 5. A projective $n$-space $P_{n}(X)$ comes equipped with a geometric filtration $P_{1}(X) \subset P_{2}(X) \subset \cdots \subset P_{n}(X)$, where $P_{1}(X)=\Sigma X$. The standard inclusion $\iota: X \rightarrow \Omega P_{n}(X)$ is the obvious adjoint. As remarked in $\S 1$, Stasheff has shown that this map is an $A_{n}$-map [19, Theorem 8.6]. Suppose, by way of contradiction, that $\iota: X \rightarrow \Omega P_{n}(X)$ is an $A_{n+1}$-map. Then by Theorem 8.4 of [19], the 
suspension, $\Sigma_{\iota}$, extends to a map $\zeta: P_{n+1}(X) \rightarrow P_{n+1}\left(\Omega P_{n}(X)\right)$. Consider the commutative diagram

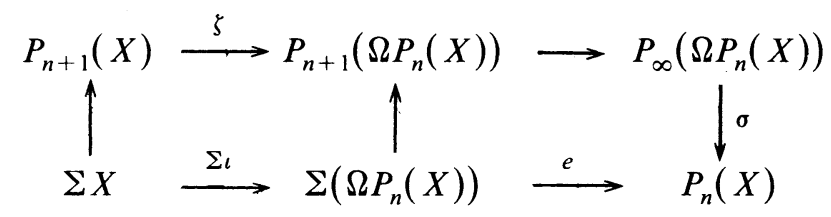

where

(i) the unlabelled maps occur in the corresponding filtrations,

(ii) the map $e$ is evaluation, $e(t, \omega)=\omega(t)$ and

(iii) $\sigma$ is an equivalence which extends $e[19$, p. 18].

Let $r: P_{n+1}(X) \rightarrow P_{n}(X)$ denote the composition in (5.1). I claim that the restriction $r_{n}=r \mid P_{n}(X): P_{n}(X) \rightarrow P_{n}(X)$ is a homotopy equivalence. Assume for the moment that this is true.

From the associated cofibration [18, Theorem 12],

$$
E_{n}(X) \stackrel{p_{n}}{\rightarrow} P_{n}(X) \rightarrow P_{n+1}(X)
$$

it follows that $r_{n} \circ p_{n} \simeq *$. However, $p_{n}$ is a quasifibration whose fiber, $X$, is nullhomotopic in $E_{n}(X)$. By exactness, it follows that $p_{n}$ (and hence $r_{n} \circ p_{n}$ ) induces a monomorphism on $\pi_{*} E_{n}(X)$. Thus

$$
\begin{aligned}
& \pi_{*} E_{n}(X)=0 \\
& \Rightarrow \tilde{H}_{*} E_{n}(X)=0, \quad \text { by the Hurewicz isomorphism theorem, } \\
& \Rightarrow \tilde{H}_{*}\left(X^{\wedge(n+1)}\right)=0, \quad \text { since } E_{n}(X) \simeq \Sigma^{n} X^{\wedge(n+1)}[18, \text { Theorem 11], } \\
& \Rightarrow \tilde{H}_{*} X=0, \quad \text { by the Kunneth formula, } \\
& \Rightarrow \pi_{1} X=0, \quad \text { since } \pi_{1}=H_{1} \text { for connected } H \text {-spaces, }
\end{aligned}
$$

and

$$
\begin{aligned}
& \pi_{*} X=0, \quad \text { again by the Hurewicz theorem, } \\
& \Rightarrow X \simeq *, \quad \text { by J. H. C. Whitehead's theorem. }
\end{aligned}
$$

This contradicts the hypothesis that $X$ is noncontractible.

To complete the proof, I must show that the map $r_{n}$ is an equivalence. Notice that in diagram 5.1, the bottom line, $e \circ \Sigma_{\iota}$, is homotopic to the given inclusion $i$; $\sum X \rightarrow P_{n}(X)$. Thus $i \circ r_{n} \simeq i$ and it suffices to prove the following.

LEMMA 5.2. Suppose that the following diagram commutes:

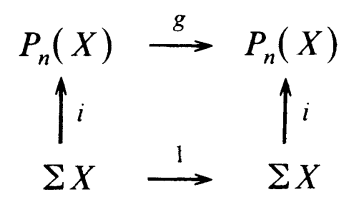

Then the map $g$ is a homotopy equivalence. 
Proof. The proof is by induction on $n$. The case $n=1$ is certainly true since $P_{1}(X)=\Sigma X$; so assume that $n>1$. I claim that there is a commutative diagram

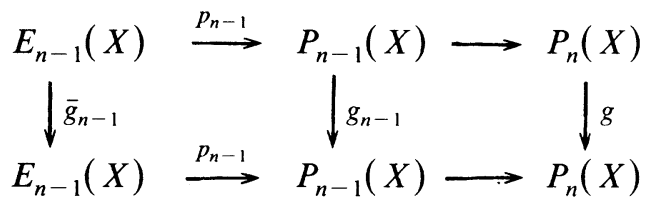

where $g_{n-1}$ also extends the identity on $\Sigma X$. To see why this is so, it is necessary to examine the correspondence between $A_{n}$-homotopies

$$
F_{i}: X^{i} \times D^{i-1} \rightarrow Y, \quad 1 \leqslant i \leqslant n, F_{1}=f
$$

and extensions $f_{i}: P_{i}(X) \rightarrow P_{i}(Y)$, of $\Sigma f$.

Just as the spaces $E_{i}(X)$ and $P_{i}(X)$ can be obtained as quotients of $X^{i+1} \times D^{i}$ and $X^{i} \times D^{i}$, respectively, the corresponding maps $\bar{f}_{i}$ and $f_{i}$ can be obtained as quotients of the homotopies $F_{i+1}$ and $1_{I} \times F_{i}$ [20, Lemma 22]. Conversely, given a map $f_{n}: P_{n}(X) \rightarrow P_{n}(Y)$, one can produce a coherent family of homotopies $F_{i}$ by taking the composition

$$
X^{i} \times D^{i-1} \stackrel{H_{i}}{\longrightarrow} \Omega P_{i}(X) \rightarrow \Omega P_{n}(X) \stackrel{\Omega f_{n}}{\longrightarrow} \Omega P_{n}(Y) \stackrel{\rho}{\longrightarrow} Y,
$$

where the $H_{i}$ 's are $A_{i}$-homotopies for $\iota$ and the map $\rho$ is an $A_{\infty}$-retraction.

In particular, if $X=Y$ and $f=1: X \rightarrow X$, we can choose $H_{i}$ and $\rho$ so that $\rho \circ H_{i}$ corresponds to the identity map on $E_{i-1}(X)$ and $P_{i}(X)$, while $\rho \circ \Omega g \circ H_{i}$ corresponds to the desired maps $\bar{g}_{i-1}$ and $g_{i}$.

Having indicated how the maps in diagram (5.3) are obtained, I shall now show that they are equivalences. By the inductive hypothesis, the map $g_{n-1}$ is an equivalence, and by diagram (5.3), we have a commutative diagram whose rows are quasifibrations.

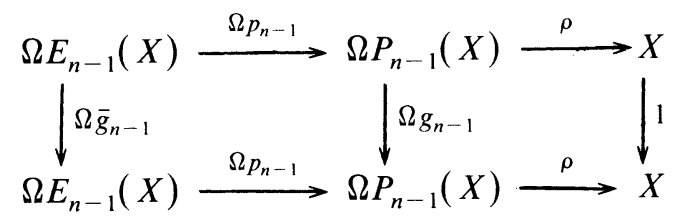

The 5-lemma, applied to the homotopy groups of this diagram, implies that $\bar{g}_{n-1}$ induces an isomorphism and, hence, is a homotopy equivalence. Now apply the 5-lemma, in homology, to diagram (5.3). It follows that $g: P_{n}(X) \hookleftarrow$ is a homology equivalence. Since $n \geqslant 2$ it is easy to see that $g$ is at least 2-connected and hence, by the Whitehead theorem, $g$ is a homotopy equivalence. 
Proof of Theorem 7. Let $f: G \rightarrow G$ be an $A_{\infty}$-map. According to Fuchs [7], there is a map of classifying spaces $B f: B G \rightarrow B G$ such that the diagram

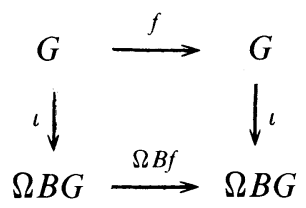

commutes, where the vertical map is a homotopy equivalence.

Since $G$ splits as a group into a product of simple Lie groups, and the functor $B$ preserves products [19], it suffices to consider the case where $G$ is also simple. Now suppose that $f$ is a power map of power $\lambda$. By naturality of the Hurewicz homomorphism, the map $B f$ must induce multiplication by $\lambda$ on the nonzero image of $\pi_{m+1} B G \rightarrow H_{m+1}(B G ; \mathbf{Q})$.

Assume for the moment that $G$ is not the exceptional Lie group $G_{2}$. Then Hubbuck [9, Theorem 2] has shown that, for a self-map $h$ of $B G$, there is an integer $k$ and an outer automorphism $\sigma$ such that $h \circ B \sigma$ induces multiplication by $k^{q}$ on $H_{2 q}(B G: \mathbf{Q})$.

Applying this result to the map $B f$ we see that, up to sign,

$$
\begin{array}{ll}
\lambda=k^{2} & \text { in dimension } 4, \\
=k^{(m+1) / 2} & \text { in dimension } m+1,
\end{array}
$$

for some integer $k$. Since $m>3$, this could only happen if $k$, and hence $\lambda$, equals 0 or \pm 1 . The possibility $\lambda=-1$ can be ruled out because this would imply that $G$ is homotopy commutative by Theorem 4. This in turn would contradict Hubbuck's classification of finite homotopy commutative $H$-spaces.

Hubbuck's results in [9] exclude the exceptional group $G_{2}$. However this case can be handled using the Adams-Mahmud theorem. By considering those maps of $H^{*}\left(B T^{2} ; \mathbf{C}\right)$ which are admissible with respect to the action of the Weyl group of $G_{2}$, one can rule out the existence of $A_{\infty}$-powers of $\lambda$ on $G_{2}$ where $|\lambda|>1$. These calculations will not be given here. The case $\lambda=-1$ can be ruled out by previous arguments.

Proof of TheOrem 8. I will again use Stasheff's characterization of $A_{n}$-maps $f$ : $X \rightarrow G$ in terms of maps $P_{n}(X) \rightarrow P_{n}(G)$. For $X=G=\operatorname{Sp}(1)$, every map is homotopic to a power map and the projective space in question is quaternionic projective $n$-space, denoted here as $P^{n}$. According to Arkowitz and Curjel [3], there is a self-map $f: P^{2} \rightarrow P^{2}$ with degree $\lambda$ in dimension 4 if and only if $\lambda(\lambda-1) \equiv 0$ mod 24. In view of these facts, the proof of Theorem 8 amounts to determining which of these maps extend to a self-map of $P^{3}$.

Let $\gamma: S^{11} \rightarrow P^{2}$ denote the quaternionic Hopf fibration. Since $P^{3}$ can be identified with the mapping cone of $\gamma$, it follows that a self-map $f$ of $P^{2}$ will extend to one of $P^{3}$ if and only if $f_{\#}(\gamma)=\lambda^{3} \gamma$ in $\pi_{11} P^{2}$. 
LEMMA 8.1. The group $\pi_{11} P^{2}$ is generated by the Hopf fibration $\gamma$ (of infinite order) and an element $g$ (of order 15). Moreover, the generator $g$ can be chosen so that if $f$ : $P^{2} \rightarrow P^{2}$ has degree $\lambda$ in dimension 4 , then $f_{\#}(g)=\lambda g$ and

$$
f_{\#}(\gamma)=\lambda^{3} \gamma+\frac{\lambda(\lambda-1)(\lambda-4)}{24} g
$$

It follows at once that $f$ extends to a self-map of $P^{3}$ iff $\lambda(\lambda-1)(\lambda-4) \equiv 0$ $\bmod 360$.

Proof of 8.1. From the long exact sequence of the $S^{3}$-fibration $\gamma$, it follows that $\pi_{11} P^{2} \approx \pi_{11} S^{11} \oplus \pi_{10} S^{3}$ where $\gamma$ generates the first summand. According to Toda [23], $\pi_{10} S^{3} \approx \mathbf{Z} / 5 \oplus \mathbf{Z} / 3$ where $\alpha_{1}$ and $\alpha_{2}$ generate the first and second summands respectively. Since the $\alpha$-family can be detected by the Adams $e$-invariant [2], the generator $g$ can be chosen to be the composition

$$
S^{11} \stackrel{g^{\prime}}{\rightarrow} S^{4} \stackrel{i}{\rightarrow} P^{2}
$$

where $g^{\prime}$ is a suspension, $e_{\mathbf{C}}\left(g^{\prime}\right)=-1 / 15$ and $i$ denotes the inclusion of the bottom 4-cell.

Now let $X=P^{3} \cup_{g} e^{12}$. Since $\pi_{11} X=0$, every self map $f$ of $P^{2}$ extends to a self map $F$ of $X$. To calculate $f_{\#}: \pi_{11} P^{2} \hookleftarrow$, I shall first compute $F_{*}: H_{12} X \hookleftarrow$ and then appeal to the following diagram

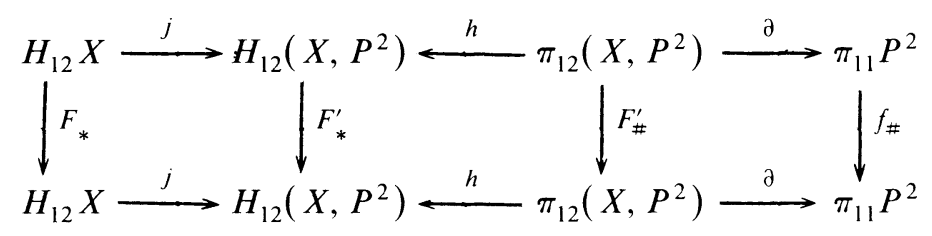

The extension $F$ is not unique since its degree can be altered by multiples of 15 on the cell attached by $g$. However it can be assumed that $F$ has been chosen so that, for independent ring generators $x \in H^{4} X$ and $y \in H^{12} X$,

$$
\begin{cases}F^{*} x^{n}=\lambda^{n} x^{n}, & n=1,2,3 \\ F^{*} y=\lambda y+c x^{3} & \text { for some integer } c .\end{cases}
$$

Let $\bar{x}^{3}$ and $\bar{y}$ denote the Kronecker duals in $H_{12} X$. It follows that in homology

$$
F_{*} \bar{x}^{3}=\lambda^{3} x^{3}+c \bar{y}, \quad F_{*} \bar{y}=\lambda \bar{y} .
$$

The generators $x$ and $y$ can be chosen so that in diagram (8.2)

$$
\partial \circ h^{-1} \circ j\left(\bar{x}^{3}\right)=\gamma, \quad \partial \circ h^{-1} \circ j(\bar{y})=g .
$$

Hence, by (8.2) it follows that $f_{\#} \gamma=\lambda^{3} \gamma+c g$ and it remains to compute the coefficient $c$. I shall use $K$-theory to do this. From the obvious inclusions

$$
P^{3} \stackrel{i_{1}}{\rightarrow} X \stackrel{i_{2}}{\leftarrow} S^{4} \cup_{g^{\prime}} e^{12}
$$


one sees that there are ring generators $z, w \in \tilde{K}(X)$ on which the Chern character takes the following values:

$$
\operatorname{ch}(z)=x+\frac{x^{2}}{12}+\frac{x^{3}}{360}-\frac{y}{15}, \quad \operatorname{ch}(w)=y .
$$

Let $F^{*} z=\sum a_{i} z^{i}+b w$ in $K(X)$. The coefficients $a_{i}$ can be determined using (8.3), (8.6) and the naturality of the Chern character. Indeed, a simple computation shows that

$$
a_{3}=\frac{\lambda^{3}-5 \lambda^{2}+4 \lambda}{360}-\frac{c}{15}
$$

Since $a_{3}$ is an integer, it follows that $\lambda^{3}-5 \lambda^{2}+4 \lambda \equiv 24 c \bmod 360$ or $c \equiv$ $\lambda(\lambda-1)(\lambda-4) / 24 \bmod 15$. This completes the proof of Lemma 8.1 and Theorem 8 .

\section{Triangles, hexagons, and jigsaw puzzles.}

Proof of Theorem 9. In part (i), $T$ will denote a fixed commuting homotopy on the group $X$. By hypothesis, $T$ satisfies property $\mathrm{P}_{1}$. In addition, $T$ is assumed to be stationary in one variable; that is, $T(x, *, t)=x=T(*, x, t)$ for all $t \in I$. A well-known result of James on retractible subspaces [25, Proposition 1.1.5] allows us to make the second assumption.

Let $n$ be a positive integer. I shall use $T$ to define a particular $A_{2}$-homotopy for the power map $x \mapsto x^{n}$ on $X$. Let $H_{n}: X \times X \times I \rightarrow X$ be the homotopy whose adjoint sends a pair $(u, v) \in X^{2}$ to the path.

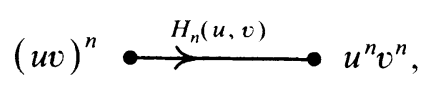

where the path $H_{n}(u, v)$ is the following:

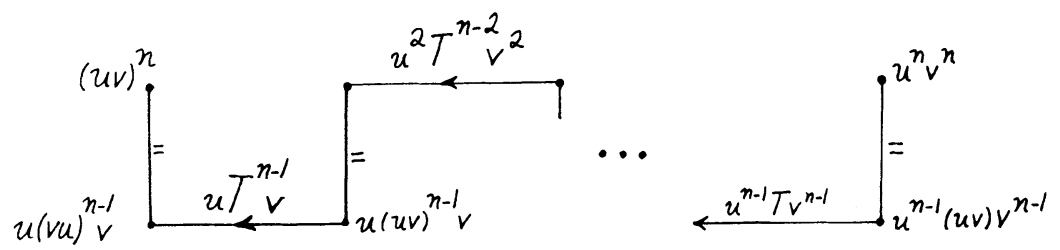

Here $T^{k}$ denotes the path from $(u v)^{k}$ to $(v u)^{k}$ given by the $k$-fold product formula,

$$
T^{k}(u, v, t)=T(u, v, t) \cdot T(u, v, t) \cdots T(u, v, t), \quad \text { with } 0 \leqslant t \leqslant 1 .
$$

Since $T$ has property $\mathrm{P}_{1}$, the homotopy $H_{n}$ can also be defined by reversing the arrows and substituting $T(v, u)$ for $T(u, v)$. 
Now let $\phi(n): X \times X \times X \times S^{1} \rightarrow X$ be the map whose adjoint sends

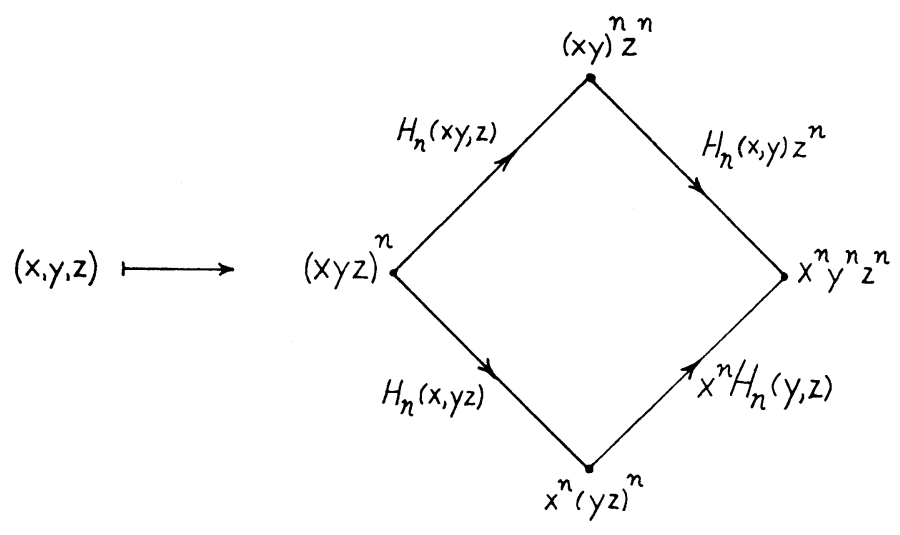

It follows from the remarks in $\S 1$ that the power map $x \mapsto x^{n}$ will be an $A_{3}$-map on $X$ if the map $\phi(n)$ can be extended to $X^{3} \times D^{2}$, or intuitively, if the above square can be filled in.

The main idea in the proof of part (i) is very simple. I shall treat the above square like a jigsaw puzzle and shall fill in as many pieces of it as I can find. The missing pieces will correspond to loops on $X$ which, a priori, cannot be filled in. Using induction on $n$, I shall show that the missing pieces come in roughly four sizes and that there are relations among the various sizes. The formula in the statement of part (i) can then be viewed as a tally of the missing pieces.

I shall now describe the missing pieces. These pieces will, of course, be maps of the form $X^{3} \times S^{1} \rightarrow X$, but it is convenient to describe the adjoints of these maps in terms of a $45^{\circ}$ right triangle. In the conversion of parameters, assume that the basepoint of the circle is sent to the right angle vertex of the triangle.

Let $\langle z \mid x y\rangle$ denote the map $X^{3} \times S^{1} \rightarrow X$ whose adjoint sends

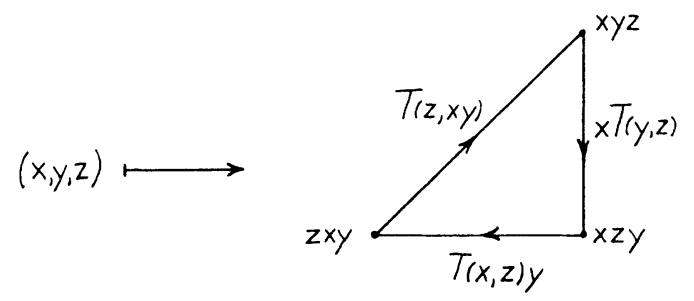

Stasheff has used triangles such as this for other purposes [19, p. 72]. Similarly, the map $\langle z y \mid x\rangle$ is defined by sending

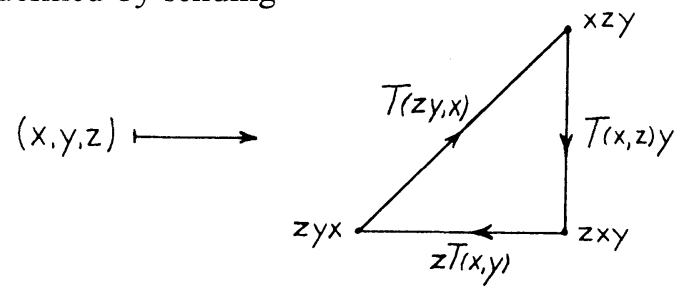


Of the 12 triangles which can be defined in this manner, we will need only four: the two just defined, $\langle x \mid y z\rangle$, and $\langle y x \mid z\rangle$.

There are universal relations among the triangles just defined which will be useful later. These relations are most clearly expressed as identities in the group $\left[X^{3}, \Omega X\right]$. Therefore, I shall first apply a transformation of the sort $\langle x \mid y z\rangle \mapsto\{x \mid y z\}$ where the latter represents the normalized adjoint of the former. In more detail, for any space $K$ and map $f: K \times S^{1} \rightarrow X$, let $L$ denote the operator such that $L(f): K \rightarrow \Omega X$ is the map which sends $k \in K$ to the loop $\theta \mapsto f(k, 0)^{-1} \cdot f(k, \theta)$, where $S^{1}$ is parameterized by $\theta, 0 \leqslant \theta \leqslant 2 \pi$.

Now use the operator $L$ to transform the triangles into based loops and denote the result with curly brackets, e.g., $\{x \mid y z\}=L\langle x \mid y z\rangle$. The hexagon $C B_{3}(T)$, defined in $\S 1$, can likewise be transformed; let $B_{3}=L C B_{3}(T)$, where the image of the basepoint occurs at the 12 o'clock position.

Proposition 9.2. In the group $\left[X^{3}, \Omega X\right]$,

(i) $\{a \mid b c\}+\{b c \mid a\}=0$, where $(a, b, c)$ denotes any permutation of $(x, y, z)$;

(ii) $B_{3}=\{x \mid y z\}-\{x \mid z y\}=\{y \mid z x\}-\{y \mid x z\}=\{z \mid x y\}-\{z \mid y x\}$.

Proof. If we reverse the orientation on $\{a \mid b c\}$, property $\mathrm{P}_{1}$ implies that the resulting loop is homotopic to $\{b c \mid a\}$. The homotopy between them, which is given by three null homotopies of the loop $L_{1}$, is illustrated in the following example,

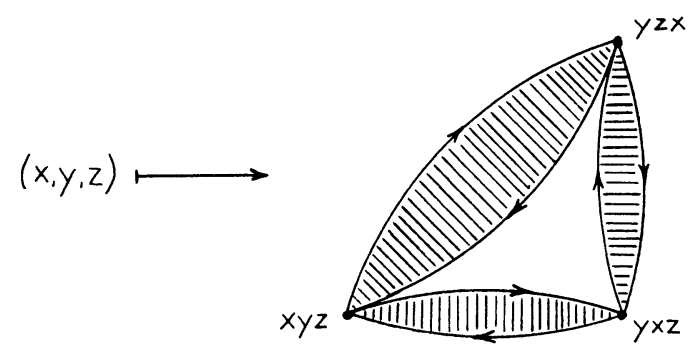

where $\langle x \mid y z\rangle$ is the outer loop and $\langle y z \mid x\rangle$ is the inner loop.

The homotopy needed for part (ii) is given by the following pictures,
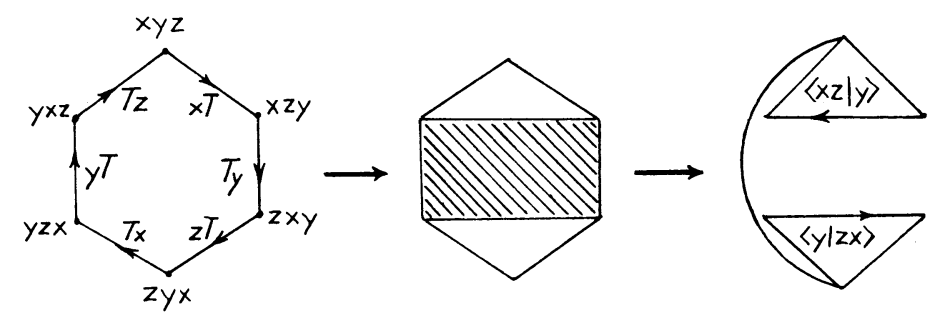

where the rectangle is filled in by the homotopy

$$
(s, t) \mapsto T(y, T(z, x, t), s) .
$$


Here $s$ is the horizontal parameter and $t$ is the vertical. Thus

$$
\begin{aligned}
& B_{3}=\{y \mid z x\}+\{x z \mid y\} \quad \text { in }\left[X^{3}, \Omega X\right] \\
& =\{y \mid z x\}-\{y \mid x z\} \quad \text { by part (i). }
\end{aligned}
$$

The other two relations are obtained by rotating the middle rectangle $\pm 60^{\circ}$.

In the formula to be proved,

$$
\Phi(n)=\frac{n(n-1)}{2} \Phi(2)+\frac{n(n-1)(n-2)}{6} B_{3},
$$

the term $\Phi(n)$ is the normalized adjoint of the map $\phi(n)$ which was defined earlier (i.e., $\Phi(n)=L \phi(n))$. As previously mentioned, this formula arises in the decomposition of the square $\phi(n)$.

Consider the case $n=2$,

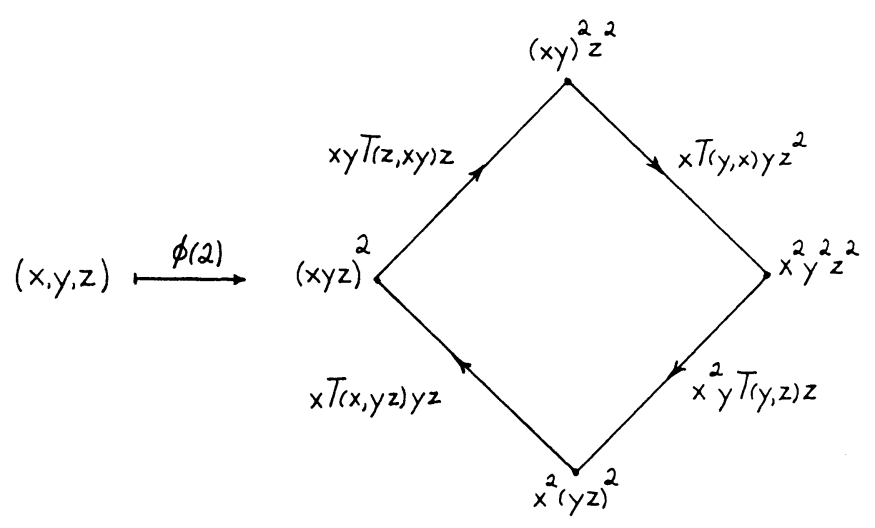

Thus, $\phi(2)$ is homotopic to

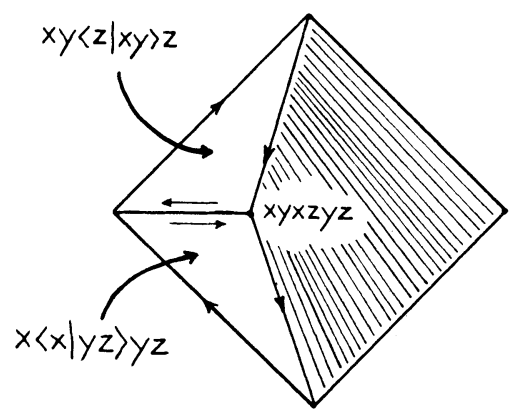

where the shaded square is filled in by the homotopy $(s, t) \mapsto x T(y, x, s) T(z, y, t) z$. This homotopy sends $(0,0)$ to the corner labelled $x y x z y z$ and it sends $(1,0)$ to the 
bottom corner, $x^{2}(y z)^{2}$. It is a simple matter to check that this homotopy agrees with $\phi(2)$ along the right-hand edges. This verification will be left to the reader.

The triangles in the second picture represent the missing pieces mentioned earlier. Each triangle can be identified by its longest side (i.e., its hypotenuse). It follows immediately from the second picture that $\phi(2) \simeq\langle z \mid x y\rangle+\langle x \mid y z\rangle$, "up to translates". This can be made precise in the group $\left[X^{3}, \Omega X\right]$ with the aid of the following technical lemma.

LEMMA 9.3. Let $h_{j}: K \rightarrow X^{I}$ for $j=1, \ldots, n$ be maps which agree at $t=0 \in I$. For each $j$, let $a_{j}, b_{j}: K \rightarrow X$ be maps whose product $a_{j} b_{j}=h_{j}$ at $t=1$. Given maps $\omega_{j}$ : $K \rightarrow \Omega X$, let $\xi$ denote the path sum

$$
\xi=\sum_{j=1}^{n} h_{j} \oplus a_{j} \omega_{j} b_{j} \ominus h_{j}
$$

and regard $\xi$ as a map $K \rightarrow X^{S^{1}}$ by identifying the endpoints of the interval $[0,3 n]$ on which the sum is defined. If $\xi / \xi_{0}$ denotes the normalization of $\xi$ (i.e., $\xi / \xi_{0}(k, \theta)=$ $\xi(k, \theta) \cdot\left[\xi(k, *)^{-1}\right)$, then

$$
\xi / \xi_{0}=\sum_{j=1}^{n} \omega_{j} \quad \text { in }[K, \Omega X]
$$

Proof. Since the summands of $\xi$ can be deformed one at a time it suffices to consider the case $n=1$. So assume that

$$
\begin{aligned}
\xi & =h \oplus a \omega b \ominus h \quad \text { with the subscripts omitted } \\
& =h \oplus a T(\omega, b, 0) \ominus h \simeq h \oplus a T(\omega, b, 1) \ominus h=h \oplus a b \omega \ominus h \\
& =h \oplus h(1) \omega \ominus h \quad \text { since } h(1)=a b \\
& \simeq h_{t} \oplus h(t) \omega \ominus h_{t} \quad \text { where } h_{t}=h \mid K \times[0, t] \\
& \simeq h(0) \omega .
\end{aligned}
$$

Since $\xi_{0}=h(0)$, the proof is complete.

$$
\text { COROllary 9.4. } \Phi(2)=\{x \mid y z\}+\{z \mid x y\} \text { in }\left[X^{3}, \Omega X\right] \text {. }
$$

This simple formula shows that the triangles such as $\langle x \mid y z\rangle$ are, in general, not homotopically trivial. Indeed, given a suitable group $X$ on which the power map $x \mapsto x^{2}$ is not $A_{3}$ (and we will later see many such examples), it follows from this formula that the triangle $\langle x \mid y z\rangle$ cannot be shrunk to a point on $X$.

In decomposing the square $\phi(n)$, the general pattern does not emerge until $n=4$. To prepare the reader for it, I shall first describe a decomposition of $\phi(3)$. 


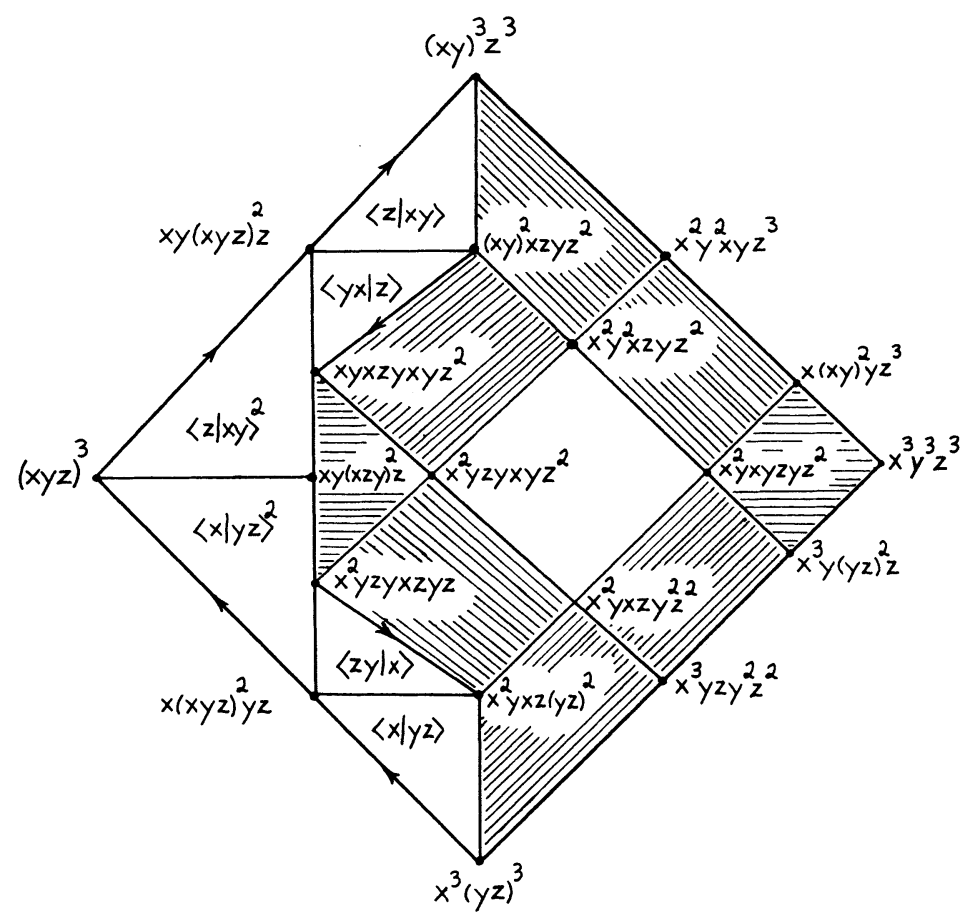

A DECOMPOSITION OF $\phi(3)$

A few remarks are in order.

1. Triangles. To keep the labels as simple as possible, I have not indicated the "coefficients" on the triangles. For example, the uppermost triangle is $(x y)^{2}\langle z \mid x y\rangle z^{2}$. These coefficients can be easily determined by checking the vertices. The power of a triangle (e.g., $\langle z \mid x y\rangle^{2}$ ) is taken with respect to the pointwise multiplication on $X$.

2. Symmetry. Notice that reflection about the equator has the effect on vertices of interchanging the letters $x$ and $z$ and then writing the resulting word backwards. This symmetry carries over to the edges and to the homotopies used in filling the squares.

3. Two dimensional homotopies. While these homotopies are quite complicated to write out, they are in principle easy to predict; each is essentially a product of its edges. The edges, in turn, represent the most economical way (in terms of the number of commutations) of getting from one vertex to the next.

The following is a list of the homotopies used in the upper half of the decomposition of $\phi(3)$. These homotopies are regarded as functions $(s, t) \in I^{2}$ with the directions noted.

$$
\begin{aligned}
& x T(y, x, s) z y x T(z, y, t) z \quad \text { in square } 1 \\
& x T(y, x, s) T(z, y x, t) y z^{2} \quad 2 \\
& x T(y, x, s) y x T(z, y, t) z^{2} \\
& x^{2} y T(y, x, s) T(z, y, t) z^{2} \quad 4 \\
& x^{2} T(y, x, s) y T(z, y, t) z^{2} \quad 5
\end{aligned}
$$




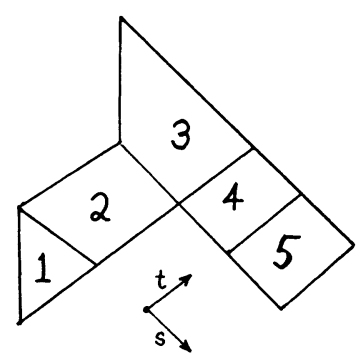

4. The hole in the middle. This missing piece has the form $x^{2} y \theta y z^{2}$ where $\theta(x, y, z)$ is the square:

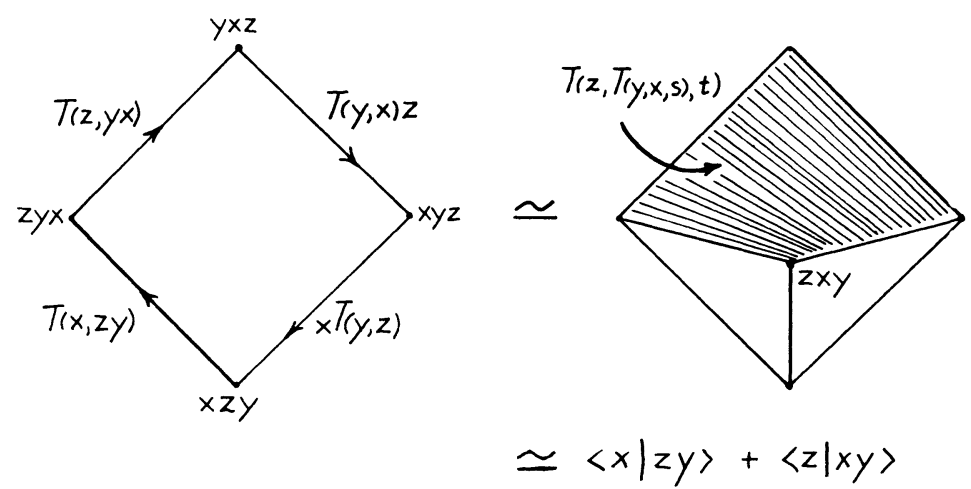

We have now accounted for all the pieces in the decomposition of $\phi(3)$. Of course one must again verify that the various homotopies agree along their common edges. Details of this sort are left to the reader.

Since $X$ is homotopy commutative, the two ways of multiplying classes in $\left[X^{3}, \Omega X\right]$ coincide. Therefore, the normalization process takes $\langle x \mid y z\rangle^{2} \mapsto 2\{x \mid y z\}$ and the tally of missing pieces is the following:

$$
\begin{aligned}
\Phi(3) & =3\{x \mid y z\}+3\{z \mid x y\}+\{y x \mid z\}+\{z y \mid x\}+\theta \\
& =3\{x \mid y z\}+3\{z \mid x y\}+\{z \mid x y\}-\{z \mid y x\} \text { by 9.2(i) } \\
& =3 \Phi(2)+B_{3} \text { by } 9.4 \text { and } 9.2(\text { ii) }
\end{aligned}
$$

Thus we have shown that the formula

$$
\Phi(n)=\frac{n(n-1)}{2} \Phi(2)+\frac{n(n-1)(n-2)}{6} B_{3}
$$

is true for $n \leqslant 3$. The induction step depends upon the following decomposition of $\phi(n)$, which contains, among other terms, a copy of $\phi(n-2)$. 


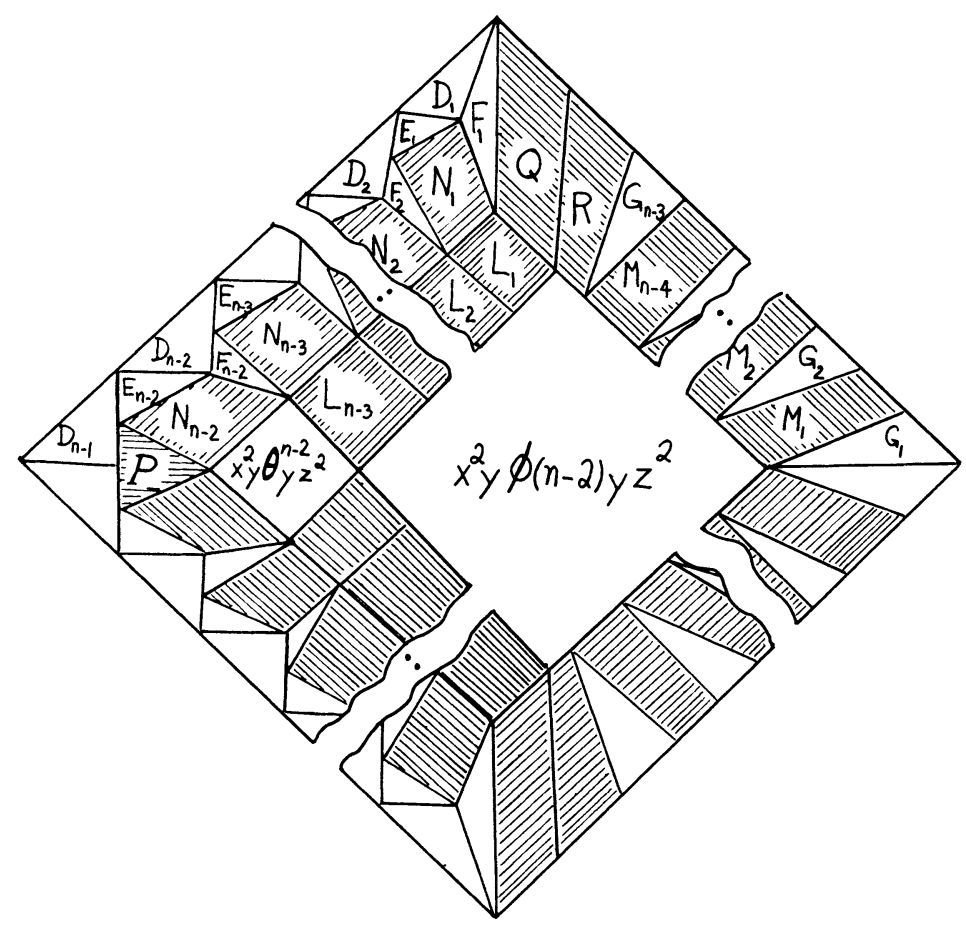

A DECOMPOSITION OF $\phi(n), n>3$

All of the pieces in this diagram have a clockwise orientation. In an attempt to keep the notation to a minimum, I have not labelled the pieces in the lower half. These regions and their corresponding homotopies can be determined using the symmetry, which was mentioned earlier, and the following description of the upper regions:

The triangles $D_{k}$ and $E_{k}$. These triangles are an immediate generalization of those which occurred in the case $n=3$.

$$
D_{k}=(x y)^{n-k}\langle z \mid x y\rangle^{k} z^{n-k}, \quad E_{k}=(x y)^{n-k-1} x\langle y x \mid z\rangle^{k} y z^{n-k} .
$$

Their "reflections" in the lower half must also be counted.

$$
D_{k}^{\prime}=x^{n-k}\langle x \mid y z\rangle^{k}(y z)^{n-k}, \quad E_{k}^{\prime}=x^{n-k} y\langle z y \mid x\rangle^{k} z(y z)^{n-k-1} .
$$

The triangles $F_{k}$ and $G_{k}$. These triangles are new. I shall show momentarily that they contribute nothing in the final tally. Here are their descriptions.

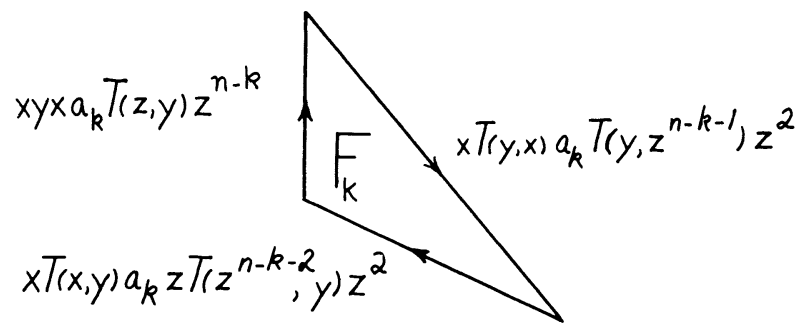

where $a_{k}=(y x)^{n-k-1}(z y x)^{k-1}$. Notice that $F_{n-2}$ collapses to a line. 


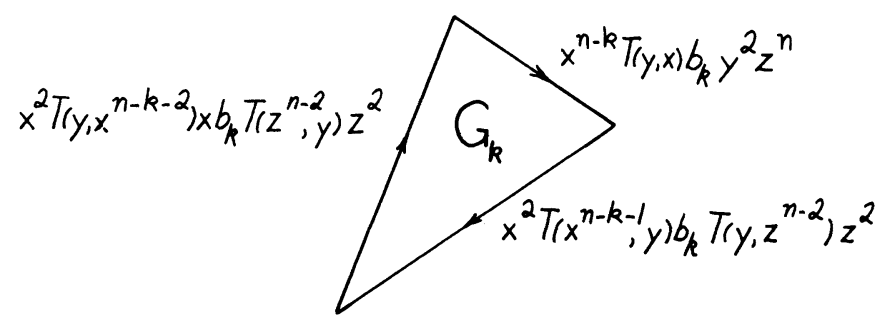

where $b_{k}=(y x)^{k-1} y^{n-k-1}$. Fortunately, these triangles do not enter into the final tally because they are cancelled out by their counterparts in the lower half of the decomposition. In more detail the $F$-family, when normalized, is of the form $F \sim\left\langle y \mid z \cdot z^{q}\right\rangle$. Similarly $G \sim\left\langle x^{q} \cdot x \mid y\right\rangle$. If $F^{\prime}$ and $G^{\prime}$ denote their reflections in the lower half, it is straightforward to see that $F^{\prime} \sim\left\langle y \mid x^{q} \cdot x\right\rangle$ and $G^{\prime} \sim\left\langle z \cdot z^{q} \mid y\right\rangle$. Thus upon normalization, these triangles cancel out by Proposition 9.2.

The two-dimensional homotopies. Each of the shaded regions in the main diagram can be viewed as a rectangle which has been rotated $-45^{\circ}$ from its usual upright position. For each triple $(x, y, z)$, the parameters $(s, t) \in I^{2}$ are mapped in the directions
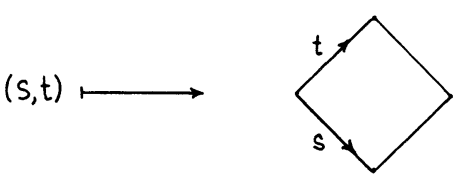

by the formulae,

$$
\begin{aligned}
& L_{k}: x^{2} y T^{n-k-2}(y, x, s) T^{k}(z, T(y, x, s), t) z^{n-k-2} y z^{2}, \\
& M_{k}: x^{2} T\left(y, x^{n-k-2}, t\right) T^{k}(y, x, s) y^{n-k-2} T\left(z^{n-2}, y, t\right) z^{2}, \\
& N_{k}: x T(y, x, s)(y x)^{n-k-2} T^{k}(z, y x, t) T\left(y, z^{n-k-2}, s\right) z^{2}, \\
& P: x T(y, x, s)(z y x)^{n-2} T(z, y, t) z, \\
& Q: x T(x, y, t(1-s)) T^{n-2}(y, x, s) T\left(z^{n-2}, y, t\right) z^{2}, \\
& R: x^{2} T(y, x, s t) T^{n-3}(y, x, s) y T\left(z^{n-2}, y, t\right) z^{2} .
\end{aligned}
$$

I realize that it is rather outrageous to ask the reader to verify that these homotopies agree along their common edges, but there seems to be no reasonable alternative. There are 21 different edges to check. For example, the edge $s=1$ of $N_{k}$ coincides with the edge $s=0$ of $L_{k}$. The corresponding homotopies restrict to the path

$$
t \mapsto x^{2} y(y x)^{n-k-2} T^{k}(z, y x, t) z^{n-k-2} y z^{2} .
$$

I assure the reader that I have checked all of the edges in the remaining cases. 
We now normalize $\phi(n)$ and express the result in terms of the pieces which occur in the main diagram. To simplify the notation, let

$$
u=\{x \mid y z\}, \quad u^{\prime}=\{x \mid z y\},
$$

and

$$
v=\{z \mid x y\}, \quad v^{\prime}=\{z \mid y x\}
$$

Then

$$
\begin{aligned}
\Phi(2) & =u+v, & & \text { by Corollary } 9.4, \\
B_{3} & =u-u^{\prime}=v-v^{\prime}, & & \text { by Proposition 9.2, }
\end{aligned}
$$

and

$$
\theta=u^{\prime}+v \quad \text { by Case } n=3, \text { Remark } 4 .
$$

By use of the counting formula $1+2+\cdots+n=n(n-1) / 2$, it follows from the decomposition of $\phi(n)$ that

$$
\Phi(n)=\frac{n(n-1)}{2}(u+v)-\frac{(n-1)(n-2)}{2}\left(u^{\prime}+v^{\prime}\right)+(n-2) \theta+\Phi(n-2) .
$$

By use of the above relations and the induction hypothesis, we have

$$
\Phi(n)=\frac{n(n-1)}{2} \Phi(2)+\frac{(n-2)(n-3)(n-4)}{6} B_{3}+W_{n}
$$

where

$$
\begin{aligned}
W_{n} & =\frac{(n-2)(n-3)}{2}(u+v)+(n-2)\left(u^{\prime}+v\right)-\frac{(n-1)(n-2)}{2}\left(u^{\prime}+v^{\prime}\right) \\
& =\frac{(n-1)(n-2)}{2}\left(v-v^{\prime}\right)+\frac{(n-2)(n-3)}{2}\left(u-u^{\prime}\right) \\
& =\frac{1}{2}[(n-1)(n-2)+(n-2)(n-3)] B_{3}=(n-2)^{2} B_{3} .
\end{aligned}
$$

Since $(n-2)(n-3)(n-4) / 6+(n-2)^{2}=n(n-1)(n-2) / 6$, we obtain the desired formula

$$
\Phi(n)=\frac{n(n-1)}{2} \Phi(2)+\frac{n(n-1)(n-2)}{6} B_{3} .
$$

This completes the proof of part (i).

Proof OF PART (ii). For each positive integer $n$, let $H_{-n}: X \times X \times I \rightarrow X$ be the homotopy which assigns to each pair $(x, y) \in X^{2}$, the path

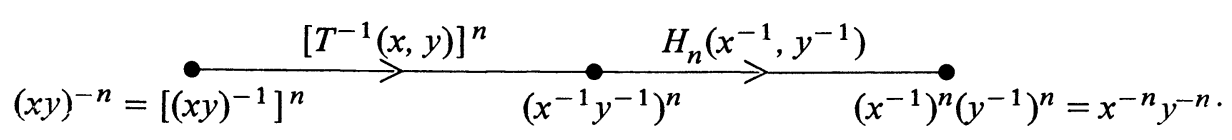

The corresponding $A_{3}$-obstruction, $\phi(-n)$, can be described in terms of $\phi(-1)$ and $\phi(n)$ by the formula

$$
\phi(-n) \simeq[\phi(-1)]^{n} \oplus \phi(n) \circ(-1 \times-1 \times-1)
$$


This formula is an easy consequence of the following picture,

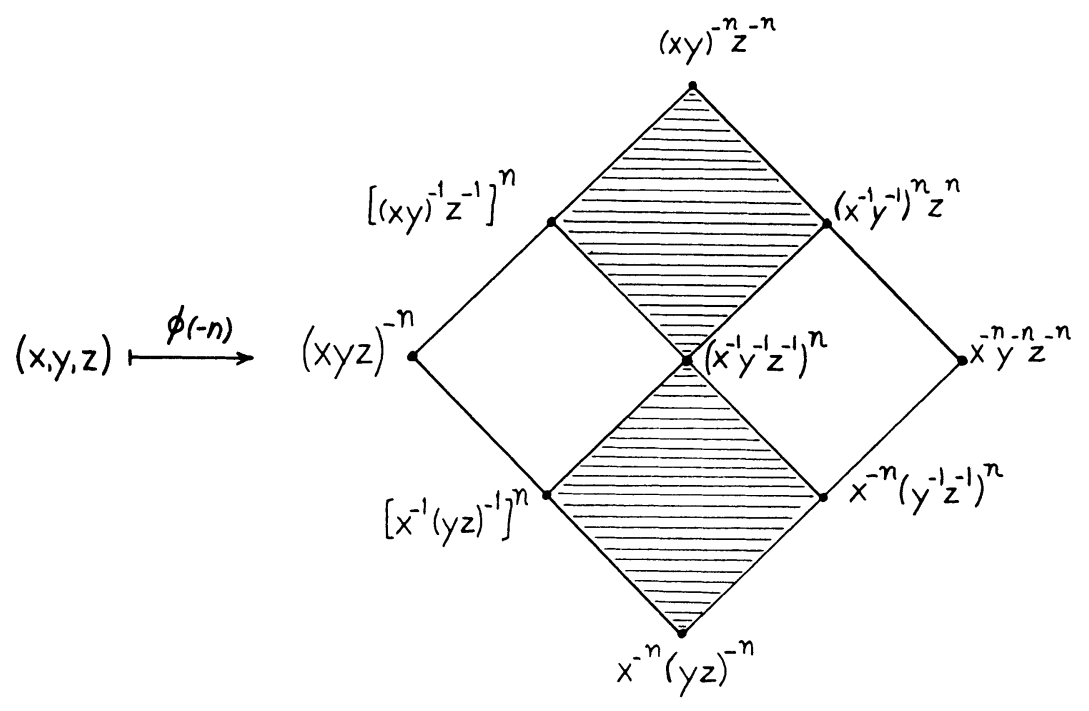

in which the uppermost square is filled by the homotopy $(s, t) \mapsto$ $H_{n}\left(T^{-1}(x, y, s), z^{-1}, t\right)$ and the bottom square is filled by the homotopy $(s, t) \mapsto$ $H_{n}\left(x^{-1}, T^{-1}(y, z, t), s\right)$.

To obtain a formula for $\Phi(-n)$, it is therefore necessary to examine the obstructions $\Phi(-1)$ and $\Phi(n) \circ(-1 \times-1 \times-1)$ in more detail. I claim that $\Phi(-1) \simeq \theta$. To see this, apply the inverse $-1: X \rightarrow X$ to the obstruction $\phi(-1)$. Since $T$ is now assumed to have properties $\mathrm{P}_{1}$ and $\mathrm{P}_{2}$, the resulting map can be deformed as follows.
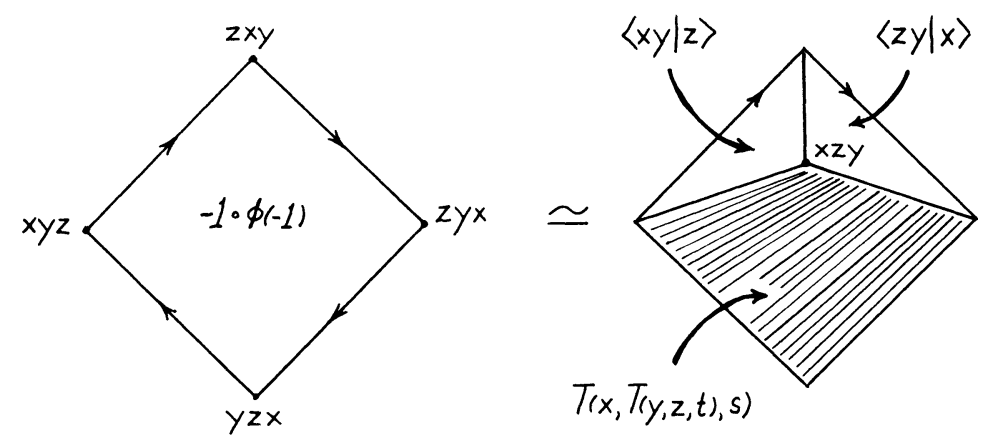

Now normalize and reverse the orientation. By Proposition 9.2, it follows that $\Phi(-1)=\{z \mid x y\}+\{x \mid z y\}=\theta$. The map $-1 \times-1 \times-1: X^{3} \rightarrow X^{3}$ induces an involution on the group $\left[X^{3}, \Omega X\right]$. To compute the value of this involution on $\Phi(n)$, I will first consider its effect on the triangles $\langle x \mid y z\rangle$ and $\langle z \mid x y\rangle$. Properties $\mathrm{P}_{1}$ and 
$\mathrm{P}_{2}$ imply that the composition $(-1) \circ\langle x \mid y z\rangle \circ(-1 \times-1 \times-1)$ is homotopic to the map

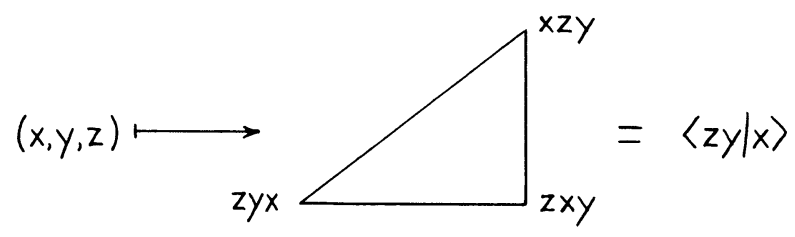

Therefore, $\{x \mid y z\} \circ(-1 \times-1 \times-1) \simeq\{x \mid z y\}$ by Proposition 9.2. A similar argument shows that $\{z \mid x y\} \circ(-1 \times-1 \times-1) \simeq\{z \mid y x\}$. Thus, in terms of the abbreviations $u, u^{\prime}, v$, and $v^{\prime}$, which were used in the proof of part (i), the involution on $\left[X^{3}, \Omega X\right]$ induced by $-1 \times-1 \times-1: X^{3} \hookleftarrow$ sends $u$ to $u^{\prime}, v$ to $v^{\prime}$, and $B_{3}$ to $-B_{3}$. It follows that in $\left[X^{3}, \Omega X\right]$,

$$
\begin{aligned}
& \Phi(-n)=n \theta+\Phi(n) \circ(-1 \times-1 \times-1) \\
& =n \theta+\left\{\frac{n(n-1)}{2} \Phi(2)+\frac{n(n-1)(n-2)}{6} B_{3}\right\} \circ(-1 \times-1 \times-1) \\
& =n\left(u^{\prime}+v\right)+\frac{n(n-1)}{2}\left(u^{\prime}+v^{\prime}\right)-\frac{n(n-1)(n-2)}{6} B_{3} \\
& =\frac{n(n+1)}{2}(u+v)+\frac{n^{2}+n}{2}\left(u^{\prime}-u\right)+\frac{n^{2}-n}{2}\left(v^{\prime}-v\right)-\frac{n(n-1)(n-2)}{6} B_{3} \\
& =\frac{n(n+1)}{2} \Phi(2)-n^{2} B_{3}-\frac{n(n-1)(n-2)}{6} B_{3} \\
& =\frac{(-n)(-n-1)}{2} \Phi(2)+\frac{(-n)(-n-1)(-n-2)}{6} B_{3} .
\end{aligned}
$$

This completes the proof of part (ii).

PROOF OF PART (iii). To prove that the elements $\Phi(2)$ and $B_{3}$ have finite order in the group $\left[X^{3}, \Omega X\right]$, it suffices, by Proposition 9.2 and Corollary 9.4, to show that the classes, $\{x \mid y z\},\{z \mid x y\}$, and so on, have finite order. Moreover, since these triangles are equivalent up to automorphisms of $\left[X^{3}, \Omega X\right]$ it is enough to consider just one, say $\{x \mid y z\}$.

Since $X$ is a connected homotopy commutative group, it is known that for any space $K,\left[K, X_{0}\right] \approx[K, X] \otimes \mathbf{Q}$ and that the kernel of the rationalization map

$$
[K, X] \stackrel{r_{*}}{\rightarrow}\left[K, X_{0}\right]
$$

consists of all elements of finite order [22, §2]. Therefore, to show that the class $\{x \mid y z\}$ has finite order it suffices to show that the rationalized triangle $\langle x \mid y z\rangle$ extends to a map $X^{3} \times D^{2} \rightarrow X_{0}$. If $(X, \mu, T)$ satisfies the hypothesis of part (iii), 
then the image of $\langle x \mid y z\rangle$ on $X_{0}$ can be decomposed as follows:

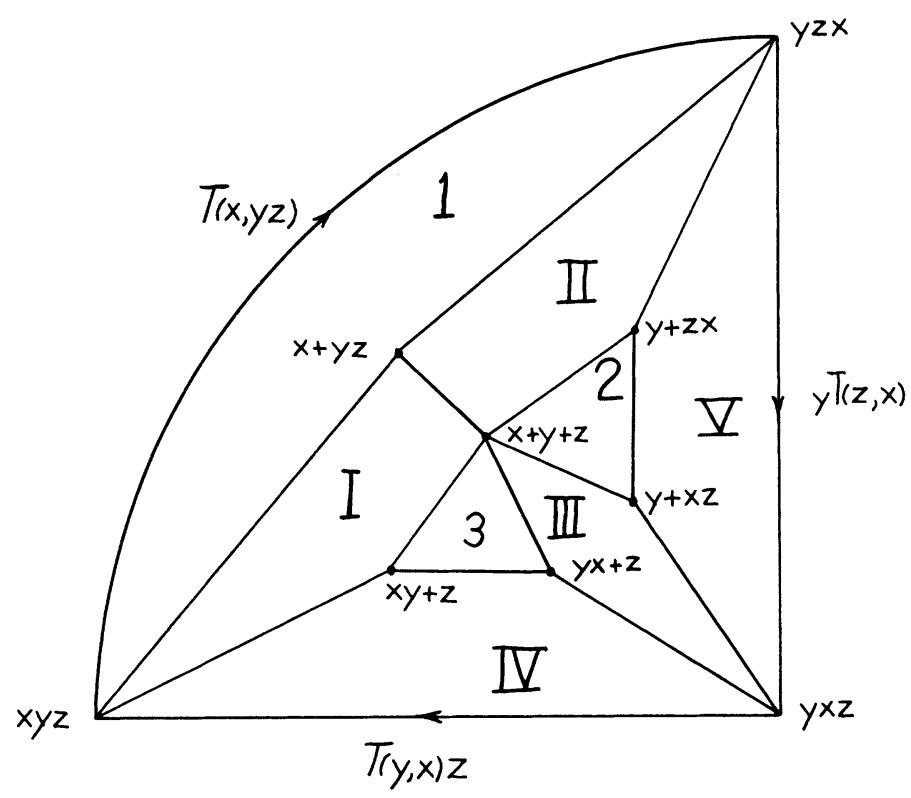

In this decomposition, the triangles labelled 1,2 and 3 can be filled in by null homotopies of $L_{3}(x, y z), y+L_{3}(z, x)$, and $L_{3}(y, x)+z$, respectively. The quadrilaterals, I, II, and III can be filled in by null homotopies of $A B_{3}(R)$, say $R_{3}(x, y, z)$, $R_{3}(y, z, x)$ and $R_{3}(y, x, z)$, respectively. The remaining two quadrilaterals can be filled in by the homotopies

$$
(s, t) \mapsto R(T(x, y, s), z, t) \text { in IV }
$$

and

$$
(s, t) \mapsto R(y, T(x, z, t), 1-s) \text { in } \mathrm{V} .
$$

It is a simple matter to check that these homotopies agree along their common edges. Again this task will be left to the reader. This completes the proof of Theorem 9.

Proof of Corollary 9.1. Assume that the power map $x \mapsto x^{n}$ is an $A_{3}$-map on ( $X, \mu)$ for some four consecutive values of $n$. These power maps must be $A_{2}$-maps as well as so Corollary 4.1 implies that $(X, \mu)$ is homotopy commutative. Let $T$ be a $C_{2}$-form on $(X, \mu)$. The assumption $[X \wedge X, \Omega X]=0$ implies that $(X, \mu, T)$ satisfies properties $\mathrm{P}_{1}$ and $\mathrm{P}_{2}$ for trivial reasons. The finiteness assumptions on $X$ imply, by Hopf's theorem, that $X_{0}$ has the homotopy type of a finite product of rational Eilenberg-Mac Lane spaces, say $K(\mathbf{Q}, \bar{n})$, where $\bar{n}=\left(2 n_{1}-1, \ldots, 2 n_{r}-1\right)$. The product multiplication on $K(\mathbf{Q}, \bar{n})$ is strictly commutative and since $H^{*}(B X ; \mathbf{Q}) \approx$ $\mathbf{Q}\left[y_{1}, \ldots, y_{r}\right]$, where $\operatorname{dim} y_{j}=2 n_{i}$, one sees that $(X, \mu)_{0}$ is $A_{\infty}$-equivalent to this product. Thus the map $L_{3}: X^{2} \times S^{1} \rightarrow X_{0}$ is defined. Since the obstruction to extending $L_{3}$ to $X^{2} \times D^{2} \rightarrow X_{0}$ lies in $\left[X \wedge X, \Omega X_{0}\right]=0$, the $C_{2}$-group $(X, \mu, T)$ satisfies property $\mathrm{P}_{3}$ as well. 
Suppose that $H^{\prime}$ is an $A_{2}$-homotopy for the power map $x \mapsto x^{n}$ on $(X, \mu)$. In the proof of Theorem 9(i), a particular homotopy $H_{n}=H_{n}(T)$ was constructed. Since [ $X \wedge X, \Omega X]=0, H^{\prime}$ can be deformed into $H_{n}$ by a homotopy which fixes their common endpoints, $(x y)^{n}$ and $x^{n} y^{n}$. It follows that in this special case the $A_{3}$ obstruction $\Phi(n)$ depends only on $n$. Hence $\Phi(n)=0$ if and only if the power map $x \mapsto x^{n}$ is an $A_{3}$ map on $(X, \mu)$.

In particular for some integer $n, \Phi(\lambda)=0$ where $\lambda=n, \ldots, n+3$. From the formula for $\Phi(\lambda)$ in Theorem 9 , it follows, using finite differences, that

$$
\sum_{k=0}^{3}(-1)^{k+1}\left(\begin{array}{l}
3 \\
k
\end{array}\right) \Phi(n+k)=B_{3}(T)=0 \text {. }
$$

A null homotopy of $B_{3}(T)$ gives rise to a $C_{3}$-form on $(X, \mu)$ and so the proof is complete.

Proof of Theorem 10. Let $T: X^{2} \times I \rightarrow X$ be a commuting homotopy on $(X, \mu)$. As explained in the proof of Theorem 9, we may assume that $T(x, *, t)=T(*, x, t)$ $=x$ for all $x \in X$ and $t \in I$. A priori, there is no reason why $T$ should satisfy properties $\mathrm{P}_{1}$ and $\mathrm{P}_{2}$. Therefore, the proof of part (i) amounts to altering $T$ by elements of $[X \wedge X, \Omega X]$ so that the resulting $C_{2}$-form will have these properties. To this end, define $\alpha: X \times X \rightarrow \Omega X$ by the formula

$$
\alpha(x, y, t)=T(x, y, t) \cdot T^{-1}(y, x, 1-t) .
$$

If $\alpha$ is null homotopic, then it is easy to see that $T$ has property $\mathrm{P}_{1}$. In the event that $\alpha$ is nontrivial, the homotopy $T$ can be altered as follows. First notice that $\alpha(x, y, t) \cdot \alpha(y, x, 1-t)=*$ for all $x, y$, and $t$. Hence $\alpha \simeq \alpha \circ \tau$, where $\tau$ is the switch map on $X^{2}$, i.e., $\tau(x, y)=(y, x)$.

Since the space $X$ is localized away from the prime 2, every class in $[X \times X, \Omega X]$ can be halved uniquely. In particular, $\alpha / 2=(1 / 2) \circ \alpha$ where $1 / 2: \Omega X \rightarrow \Omega X$ denotes the corresponding power map. The formulae $\alpha / 2+\alpha / 2=\alpha$ and $\alpha / 2 \circ \tau$ $=\alpha / 2$ are easily seen to hold in $[X \times X, \Omega X]$. Moreover, $\alpha / 2$ may be represented by a map (with the same name) which is constant in $X \vee X$. If $T^{\prime}$ is defined by the formula

$$
T^{\prime}(x, y, t)=T(x, y, t) \cdot \alpha / 2(x, y, 1-t),
$$

it is straightforward to check that the corresponding loop $\alpha^{\prime}$ is null homotopic. Hence $T^{\prime}$ satisfies property $\mathrm{P}_{1}$.

To achieve property $\mathrm{P}_{2}$, I shall again alter $T$ if necessary by removing one-half of the unwanted loop. In more detail, assume that $T$ is a commuting homotopy which is stationary on the wedge $X \vee X$ and which satisfies property $\mathrm{P}_{1}$. Define $\beta: X \times X \rightarrow$ $\Omega X$ by the formula

$$
\beta(x, y, t)=T(x, y, t) \cdot T\left(y^{-1}, x^{-1}, t\right) .
$$

Notice that $\beta$ is constant on $X \vee X$ and that, by property $\mathrm{P}_{1}, \beta \simeq-\beta \circ \tau$. If $\tau^{\prime}(x, y)=\left(y^{-1}, x^{-1}\right)$, it is straightforward to check that $\beta \circ \tau^{\prime} \simeq \beta$. The class $\beta / 2$ inherits these same properties. Hence, if $T^{\prime \prime}$ is given by the formula

$$
T^{\prime \prime}(x, y, t)=T(x, y, t) \cdot \beta / 2(x, y, 1-t)
$$


it is easy to see that the corresponding $\beta^{\prime \prime}$ is trivial. Thus $T^{\prime \prime}$ has property $\mathrm{P}_{2}$. Moreover, since $\beta / 2 \simeq-\beta / 2 \circ \tau, T^{\prime \prime}$ also satisfies property $\mathrm{P}_{1}$. This completes the proof of part (i) of Theorem 10 .

If $G$ is isomorphic to a product of groups $G_{1} \times \cdots \times G_{m}$, then $G$ is homotopy commutative if and only if each of the factors $G_{i}$ is. Moreover, if each $G_{i}$ has a commuting homotopy $T_{i}$ with properties $\mathrm{P}_{1}, \mathrm{P}_{2}$, and $\mathrm{P}_{3}$ then the product $C_{2}$-form $T=T_{1} \times \cdots \times T_{m}$ also has these properties. These considerations and the classification theorem for Lie groups imply that it is enough to prove Theorem 10(ii) for the case where $G$ is a simply connected simple Lie group.

Lemma 10.1. Let $G=\operatorname{SU}(n), \operatorname{Sp}(n)$, or $\operatorname{Spin}(n)$ where $n \geqslant 2,3$, or 7 respectively. If $l$ is a set of primes at which $G$ is homotopy commutative, then

(a) $G$ is l-equivalent to a product of spheres, and

(b) there is a $C_{2}$-form $T$ on $G_{(l)}$ with properties $\mathrm{P}_{1}, \mathrm{P}_{2}$, and $\mathrm{P}_{3}$.

In other words, part (a) asserts that $l$ must be a set of regular primes for $G$. The proof of the first part uses Bott's results on Samelson products in the classical groups [4] and the following result of Serre [17] on regular primes.

THEOREM 10.2. A prime $p$ is a regular prime for a group $G$ if $p \geqslant n$ and $G=\mathrm{SU}(n)$, or if $p \geqslant 2 n$ and $G=\operatorname{Sp}(n), \operatorname{Spin}(2 n+1)$, or $\operatorname{Spin}(2 n+2)$.

Let $x_{i} \in \pi_{2 i-1} \mathrm{SU}(n)$ denote a generator. Bott showed that the order of the Samelson product $\left\langle x_{i}, x_{j}\right\rangle$ in $\pi_{*} S U(n)$ is a nonzero multiple of

$$
(i+j-1) ! /(i-1) !(j-1) ! \quad \text { if } 1<i, j \leqslant n \text { and } n \leqslant i+j-1 .
$$

If $p$ is a prime less than $2 n$ then it is easy to check that $p$ divides at least one of these orders. By the definition of a Samelson product, it follows than $\operatorname{SU}(n)$ is not homotopy commutative at such a prime. Hence for $G=\mathrm{SU}(n)$, the set $l$ contains only primes $p \geqslant 2 n$, which are regular by Theorem 10.2 .

The proof in the symplectic case is similar. Let $y_{i} \in \pi_{4 i-1} \mathrm{Sp}(n)$ denote a generator. Here Bott showed that the order of the Samelson product $\left\langle y_{i}, y_{j}\right\rangle$ is a nonzero multiple of $\frac{1}{2}(2 i+2 j-1) /(2 i-1) !(2 j-1)$ ! if $1 \leqslant i, j \leqslant n$ and $n \leqslant i+j-1$. A careful examination of these orders shows that if $p$ is an odd prime less than $4 n$, then, with just one exception, $p$ divides at least one of these orders. The lone exception occurs when $n=2$ and $p=3$. This is why $\operatorname{Sp}(2)$ is excluded from the statement of Lemma 10.1. For the groups $\operatorname{Sp}(n)$, where $n>2$, part (a) now follows by Serre's result.

We now consider the spinor groups. Away from the prime 2, Friedlander [6, Theorem 2.1] has shown that there is an $A_{\infty}$-equivalence $\operatorname{Sp}(n) \approx \operatorname{Spin}(2 n+1)$. Thus the case $G=\operatorname{Spin}(2 n+1)$ has already been verified.

If $i, j<n$, then the product $\left\langle y_{i}, y_{j}\right\rangle$ factors through $\operatorname{Sp}(n-1)$, where it also has finite order. Therefore Friedlander's equivalence and the standard inclusions $\operatorname{Spin}(2 n-1) \rightarrow \operatorname{Spin}(2 n) \rightarrow \operatorname{Spin}(2 n+1)$ imply that, for $n>3, \operatorname{Spin}(2 n)$ is not homotopy commutative at any odd prime $p<4 n-4$. This fact, Theorem 10.2, and Hubbuck's 2-primary result complete the proof of part (a) of Lemma 10.1.

The proof of part (b) uses induction on $n$. Let $X_{n}$ denote the localization of $\mathrm{SU}(n)$ at a set of primes $l$ at which this group is homotopy commutative. 
Since $\mathrm{SU}(2) \simeq S^{3}$, the group $\left[X_{2} \wedge X_{2}, \Omega X_{2}\right] \approx \pi_{7} S_{(l)}^{3}=0$. Here the homotopy group is zero because the prime $2 \notin l$ and $\pi_{7} S^{3} \approx \mathbf{Z} / 2$ [23]. Thus, up to homotopy, there is only one $C_{2}$-form on $X_{2}$ and it has properties $\mathrm{P}_{1}, \mathrm{P}_{2}$, and $\mathrm{P}_{3}$.

Throughout the induction step assume that all spaces and maps have been localized at a set of primes $l$, at which $\mathrm{SU}(n)$ is homotopy commutative. It follows from the proof of part (a) that $\mathrm{SU}(n-1)$ is also homotopy commutative at $l$. Therefore, by induction, assume that there is a $C_{2}$-form $T_{n-1}$ on $X_{n-1}$ with properties $\mathrm{P}_{1}, \mathrm{P}_{2}$, and $\mathrm{P}_{3}$.

By part (a) there is a homotopy equivalence $\psi: X_{n} \rightarrow X_{n-1} \times S^{2 n-1}$ which, on the first factor, is a retraction for the usual inclusion $X_{n-1} \rightarrow X_{n}$, and which, on the second factor, is the principal $X_{n-1}$-fibration.

Since the prime $2 \notin l$, there is a homotopy commutative multiplication $m$ on $S^{2 n-1}$, by Adams [1, p. 54]. Let $T$ be a commuting homotopy on the $H$-space $\left(S^{2 n-1}, m\right)$.

Let $\mu_{n}$ and $\mu_{n-1}$ denote the group multiplications on $X_{n}$ and $X_{n-1}$, respectively. Consider the map

$$
\mu_{n}^{\prime}=\psi^{-1} \circ\left(\mu_{n-1} \times m\right) \circ(\psi \times \psi)
$$

where $\psi^{-1}: X_{n-1} \times S^{2 n-1} \rightarrow X_{n}$ represents a homotopy inverse for $\psi$ and which restricts on the first factor to the inclusion $X_{n-1} \rightarrow X$. Notice that $\mu_{n}^{\prime}$ and $\mu_{n}^{\prime} \circ \tau$ are homotopic. A homotopy between them is given by

$$
T_{n}^{\prime}=\psi^{-1} \circ\left(T_{n-1} \times T\right) \circ(\psi \times \psi) .
$$

Let $d=\mu_{n} \cdot\left(\mu_{n}^{\prime}\right)^{-1}: X_{n} \times X_{n} \rightarrow X_{n}$ be the difference map, where the indicated group operations are performed using the multiplication $\mu_{n}$. The map $d$ has two important features. First, $d \mid X_{n-1} \times X_{n-1}=*$, since $\mu_{n}$ and $\mu_{n}^{\prime}$ agree on this subspace. Secondly, $d$ and $d \circ \tau$ are homotopic because $\mu_{n}$ and $\mu_{n}^{\prime}$ have this property. Since $X_{n-1}^{2}$ is a retractible subspace of $X_{n}^{2}$, a homotopy $D$ between $d$ and $d \circ \tau$ can be chosen to be constant on $X_{n-1}^{2}$.

Now let $T_{n}=D \cdot T_{n}^{\prime}: X_{n}^{2} \times I \rightarrow X_{n}$. Clearly $T_{n}$ is a $C_{2}$-form on $\left(X_{n}, \mu_{n}\right)$. Since $T_{n}$ evidently agrees with $T_{n-1}$ on $X_{n-1}^{2} \times I$, the deviations of $T_{n}$ from properties $\mathrm{P}_{1}$ and $\mathrm{P}_{2}$ factor through the group $\left[X_{n} \wedge X_{n} / X_{n-1} \wedge X_{n-1}, \Omega X_{n}\right]$. In addition, the deviation of $T_{n}$ from property $\mathrm{P}_{3}$ factors through the rationalization of this group. But the quotient space $X_{n} \wedge X_{n} / X_{n-1} \wedge X_{n-1}$ is $2 n+1$ connected and so the group in question is finite. Thus $T_{n}$ has property $\mathrm{P}_{3}$ and its deviation from properties $\mathrm{P}_{1}$ and $\mathrm{P}_{2}$ are of finite order.

Recall from the proof of part (i) of Theorem 10 how $T_{n}$ can be altered, by subtraction of one-half the deviations (there denoted by $\alpha$ and $\beta$ ), so that the resulting $C_{2}$-form has properties $P_{1}$ and $P_{2}$. Due to the finiteness of the deviations and the rational nature of property $\mathrm{P}_{3}$, it is clear that these alterations can be carried out on $T_{n}$ while preserving property $\mathrm{P}_{3}$.

This completes the proof of Lemma 10.1 for the special unitary groups. The proof just given can obviously be adapted to the symplectic groups once the first case, $\mathrm{Sp}(3)$, has been established. 
To this end, let $l$ be a set of primes at which $\mathrm{Sp}(3)$ is homotopy commutative and for $n \leqslant 3$ let $X_{n}$ denote the localization of $\operatorname{Sp}(n)$ at $l$. Each prime in $l$ is larger than 12 (by the proof of part (a)) and so each such prime is regular for $\operatorname{Sp}(n), n \leqslant 3$. We previously saw that $X_{1}$ has a $C_{2}$-form with the required properties. Hence the same is true for $X_{2}$ and $X_{3}$, by the induction step in the $\mathrm{SU}(n)$ proof. It is the aforementioned regularity that allows us to use this induction step.

By Friedlander's equivalence, Lemma 10.1 also holds for $G=\operatorname{Spin}(2 n+1)$. The group $\operatorname{Spin}(2 n)$ can then be handled by using the method in the induction step and the principal fibration $\operatorname{Sp}(2 n-1) \rightarrow \operatorname{Sp}(2 n) \rightarrow S^{2 n-1}$.

It remains to prove Theorem 10(ii) for the groups $\mathrm{Sp}(2)$, and the exceptional groups $G_{2}, F_{4}, E_{6}, E_{7}$, and $E_{8}$. Let $X=G_{(l)}$ where $G$ is one of these groups and $l$ is a set of primes at which $G$ is homotopy commutative. Since $2 \notin l$, it follows from part (i) that there is a $C_{2}$-form $T$ on $X$ with properties $\mathrm{P}_{1}$ and $\mathrm{P}_{2}$.

I do not know if, in general, there is a $C_{2}$-form on $X$ with property $\mathrm{P}_{3}$. Fortunately there is a way to get around this obstacle. A tedious, but straightforward, cohomology calculation shows that the group $[X \wedge X \wedge X, \Omega X]$ is rationally trivial. Since the classes $\Phi(2)$ and $B_{3}$ live in this group, they must have finite order. This completes the proof of part (ii).

The proof of part (iii) requires the following calculations:

$$
\begin{aligned}
{\left[Y_{p} \wedge Y_{p}, Y_{p}\right] } & \approx \pi_{2 p-4} S_{(p)}^{p-2}=0, \\
{\left[Y_{p} \wedge Y_{p}, \Omega Y_{p}\right] } & \approx \pi_{2 p-3} S_{(p)}^{p-2}=0, \\
{\left[Y_{p} \wedge Y_{p} \wedge Y_{p}, \Omega Y_{p}\right] } & \approx \pi_{3 p-5} S_{(p)}^{p-2}=\mathbf{Z} / p,
\end{aligned}
$$

which can be found in Toda's book [23]. The first of these implies that $Y_{p}$ is homotopy commutative; the second implies that the $C_{2}$-form $T$ is unique; and the third shows that the $A_{3}$-obstructions may be nonzero.

In this special case, there is one more relation among the classes defined in Theorem 9; namely, $\Phi(2)=B_{3}(T)$. To see this, recall the formulae

$$
\begin{array}{cl}
\Phi(2)=\{x \mid y z\}+\{z \mid x y\} & \text { from Corollary 9.4, } \\
B_{3}(T)=\{x \mid y z\}-\{x \mid z y\} & \text { from Proposition 9.2. }
\end{array}
$$

On the smash product $Y_{p} \wedge Y_{p} \wedge Y_{p} \simeq_{p} S^{3 p-6}$, the involution which switches the first and third factors has degree -1 . This involution also interchanges $\{z \mid x y\}$ and $\{x \mid z y\}$. The relation $\Phi(2)=B_{3}(T)$ follows. This relation simplifies the formula for $\Phi(n)$ in Theorem 9, $i$ to the following

$$
\Phi(n)=\frac{n^{3}-n}{6} B_{3}(T)
$$

Since the class $B_{3}(T)$ can have order at most $p$, this shows that the condition $n^{3}-n \equiv 0 \bmod p$ is sufficient for the map $y \mapsto y^{n}$ to be an $A_{3}$-map. On the other hand, a $\mathcal{P}^{1}$ argument on $H^{*}\left(P_{3}\left(Y_{p}\right) ; \mathrm{Z} / p\right)$ shows that this condition is indeed necessary. 
6. Concluding remarks and questions. Let me take this opportunity to point out an error in part I. In the list of examples in Table 7, I claimed that $\mathrm{Sp}(2)$ is not homotopy commutative at the prime $p=3$. This is false. The proof that $\operatorname{Sp}(2)$ is homotopy commutative at $p=3$ will be given elsewhere. The reader may have noticed how this group was handled differently in the proof of Theorem 10 .

There are still many questions about power maps yet to be answered.

Question 11. Let $G$ be one of the classical Lie groups. Does the classification of $A_{3}$-power maps on $G$ take the form that the special case $G=\operatorname{Sp}(1)$ in Theorem 8 suggests? In other words, are $A_{3}$-powers on $G$ classified as common solutions to $\lambda(\lambda-1) \equiv 0 \bmod N$, from Theorem 4 , and some cubic congruence?

QUESTION 12. Is there a "calculus of preferred paths" on a homotopy commutative group which would allow one to formalize the proof of Theorem 9?

Obviously some sort of formalism is needed to carry out a similar analysis of $A_{4}$-power maps, where the obstructions such as $A B_{4}$ and $C B_{4}$ are much harder to draw!

QUESTION 13. Is there a $C_{3}$-topological group on which the power map $x \mapsto x^{n}$ is not $A_{3}$ for some integer $n$ ?

An answer to this question might serve two purposes. It could determine whether or not Corollary 9.1 is the best possible analogue of Corollary 4.1. It might also point to other basic relations besides those stated in Proposition 9.2.

It would be interesting to find the best possible mod $p$ analogue of Theorem 7 . This would help to identify those homotopy theoretic properties which are cruical to this result. The following seems plausible.

Conjecture 14. Let $X$ be a finite loop space which is 1-connected, p-local, and of rank $\geqslant 2$. Assume that $B X^{\wedge}$ contains no nontrivial retracts. If the power map $x \rightarrow x^{\lambda}$ is an $A_{\infty}$-map for some integer $\lambda$, then $|\lambda|=0$ or 1 .

\section{REFERENCES}

1. J. F. Adams, The sphere, considered as an H-space mod p, Quart. J. Math. Oxford. Ser. (2) 12 (1961), 52-60.

2. On the groups $J(X)$. IV, Topology 5 (1966), 21-71.

3. M. Arkowitz and C. R. Curjel, On the maps of H-spaces, Topology 6 (1967), 137-148.

4. R. Bott, A note on the Samelson product in the classical groups, Comment. Math. Helv. 34 (1960), 249-256.

5. S. Feder and S. Gitler, Mappings of quaternionic projective space, Bol. Soc. Mat. Mexicana 18 (1973), 33-37.

6. E. M. Friedlander, Exceptional isogenies and the classifying spaces of simple Lie groups, Ann. of Math. (2) 101 (1975), 510-520.

7. M. Fuchs, Verallgemeinerte Homotopie-homomorphismen und klassifizierende raume, Math. Ann. 161 (1965), 197-230.

8. J. R. Hubbuck, On homotopy commutative H-spaces, Topology 8 (1969), 119-126.

9. H_ Homotopy homomorphisms of Lie groups, London Math. Soc. Lecture Notes Ser. No. 11, Cambridge Univ. Press, 1974, pp. 33-41.

10. D. M. Kan, A relation between $C W$ complexes and free c.s.s. groups, Amer. J. Math. 81 (1959), 512-520 
11. A. L. Liulevicius, The factorization of cyclic reduced powers by secondary cohomology operations, Proc. Nat. Acad. Sci. U.S.A. 46 (1960), 978-981.

12. C. A. McGibbon, Multiplicative properties of power maps. I, Quart. J. Math. Oxford (2) 31 (1980), $341-350$.

13. , Generalized Samelson products in the unitary group, Notices Amer. Math. Soc. 26 (1979), Abstract 770-G9, A-588.

14. J. W. Milnor, Construction of universal bundles. I, Ann. of Math. (2) 63 (1956), 272-284.

15. J. C. Moore, Algèbres d'Eilenberg Maclane et homotopie, Séminaire H. Cartan, Paris, 1955.

16. $325-350$. , Some applications of homology theory to homotopy problems, Ann. of Math. (2) 58 (1953),

17. J. P. Serre, Groupes d'homotopie et classes de groupes abélians, Ann. of Math. 58 (1953), 258-294.

18. J. D. Stasheff, Homotopy associativity of H-spaces. I, II, Trans. Amer. Math. Soc. 108 (1963), 275-292; ibid. 108 (1963), 293-312.

19. __ H-spaces from a homotopy point of view, Lecture Notes in Math., vol. 161, Springer, Berlin, 1970.

20. M. Sugawara, A condition that a space is group-like, Math. J. Okayama Univ. 6 (1957), 109-129.

21. On the homotopy-commutativity of groups and loop spaces, Mem. Coll. Sci. Univ. Koyoto Ser. A 33 (1960/61), 257-269.

22. D. Sullivan, Geometric topology. I: Localization, periodicity, and Galois symmetry, M.I.T. Notes (1970).

23. H. Toda, Composition methods in homotopy groups of spheres, Ann. of Math. Studies, no. 49, Princeton Univ. Press, Princeton, N. J., 1962.

24. F. D. Williams, Higher homotopy-commutativity, Trans. Amer. Math. Soc. 139 (1969), 191-206.

25. A. Zabrodsky, Hopf spaces, North-Holland Mathematics Studies, no. 22, North-Holland, Amsterdam, 1976.

DePartment of Mathematics, Indiana University, Bloomington, Indiana 47405

Current address: Department of Mathematics, University of Kentucky, Lexington, Kentucky 40506 